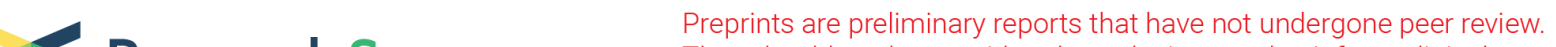 $\begin{array}{ll}\text { Research Square } & \text { They should not be considered conclusive, used to inform clinical practice, } \\ \text { or referenced by the media as validated information. }\end{array}$
}

\section{PUMILIO Proteins in Colorectal Cancer: Tumor Growth Promoting Function and Potential as Therapeutic Targets}

\section{Yuanyuan Gong}

Shanghai Institute for Advanced Immunochemical Studies

\section{Zukai Liu}

Shanghai Institute for Advanced Immunochemical Studies

\section{Yihang Yuan}

Shanghai Jiao Tong University School of Medicine (SJTU-SM), Shanghai, China

\section{Zhenzhen Yang}

Shanghai Institute for Advanced Immunochemical Studies

Jiawei Zhang

Shanghai Institute for Advanced Immunochemical Studies https://orcid.org/0000-0002-5782-5543

Qin Lu

Shanghai Jiao Tong University

\section{Wei Wang}

ShanghaiTech University

\section{Chao Fang}

Shanghai Jiao Tong University

\section{Haifan Lin}

Yale Stem Cell Center, Yale University School of Medicine, New Haven, CT 06510

https://orcid.org/0000-0002-5374-9491

Sanhong Liu ( $\square$ liush@shanghaitech.edu.cn )

Shanghai Institute for Advanced Immunochemical Studies https://orcid.org/0000-0002-8695-0343

\section{Article}

Keywords: Pumilio, colorectal cancer, p21, RNAi therapy

Posted Date: February 11th, 2021

DOl: https://doi.org/10.21203/rs.3.rs-199046/v1

License: (c) (1) This work is licensed under a Creative Commons Attribution 4.0 International License. Read Full License 
Version of Record: A version of this preprint was published at Nature Communications on March 25th, 2022. See the published version at https://doi.org/10.1038/s41467-022-29309-1. 


\section{Abstract}

PUMILIO (PUM) proteins belong to the highly conserved PUF family post-transcriptional regulators involved in diverse biological processes. However, their function in carcinogenesis remains under explored. Here, we found that the expression of Pum1 and Pum2 are increased in clinical colorectal cancer (CRC). Intestine-specific knockout of Pum1 and Pum2 significantly inhibited the progression of colitis associated cancer in the AOM/DSS model. Knockout or knockdown of Pum1 and/or Pum2 resulted in a significant decrease in the tumorigenicity. In addition, delayed G1/S transition was observed. We identified p21/Cdkn1a as direct target of PUM1, and abrogation of the PUM1 binding site in p21 resulted in decreased tumor cell growth as well as delayed G1/S transition. Furthermore, intravenous injection of nanoparticle-encapsulated anti-Pum1 and Pum2 siRNAs reduced colorectal tumor growth in murine orthotopic colon cancer models. These findings reveal a tumor growth promoting role of PUM proteins in CRC and its potential as therapeutic targets.

\section{Introduction}

According to a global epidemiological analysis in 2018 , colorectal cancer (CRC) accounts for $10 \%$ of all cancers, ranking third, with its mortality rate ranked second ${ }^{1}$. Although genetic alterations such as $A P C$, TP53, KRAS and key signaling pathways including Wnt, RAS-MAPK, PI3K drive CRC have been extensively studied ${ }^{2,3}$, the incidence and mortality of CRC is still at the forefront of all types of cancer and have been on the rise. There is still a pressing need to investigate molecular mechanisms underlying the pathogenesis of CRC for early detection, diagnosis and targeted therapy. Recent studies have shown that post-transcriptional regulation mediated by RNA binding proteins plays an important role in the initiation and development of CRC. Moreover, a large number of studies have shown the abnormal expression of RNA binding proteins in CRC cells ${ }^{4,5}$. However, the regulation at the post-transcriptional levels mediated by RNA binding proteins, emerging important, remains much less investigated.

PUM proteins belong to the highly conserved PUF (Pumilio-Fem3-binding Factor) family of RNA-binding proteins ${ }^{6}$. Mammalians have two PUM homologs, PUM1 and PUM2, that bind target transcripts through the PRE representing by an eight-nucleotide sequence motif (UGUANAUA). In most cases, PUM proteins bind to the 3'UTR of target mRNAs, and recruit partner proteins to reduce the stability of the mRNAs or inhibit the translation of proteins ${ }^{7-9}$. Many PUF target genes have been identified for their important roles in embryonic development, body axis formation, neurogenesis, spermatogenesis, and self-renewal of stem cells ${ }^{6,10-19}$. Recently, PUM proteins have emerged as important regulators in cancer. Several reports found PUM proteins are required for cancer cell growth, suggested that Pum function as an oncogene ${ }^{20-23}$. In addition, PUM1 and PUM2 growth-promoting activities are mediated by cell cycle related genes in leukemic cells ${ }^{20}$. By contrast, another angle on this debate suggested that PUM repression of the oncogenes is circumvented in multiple cancer cells including CRC ${ }^{24,25}$. Another study in CRC showed PUM hyper- or hypoactivity would lead genome instability ${ }^{24,25}$. These contrasting 
proposals indicated that the PUM function in colorectal oncogenesis and underlying mechanism remain largely elusive.

Here, we report the up-regulated expression and cancer-promoting function of Pum 1 and Pum 2 in clinical CRC samples and cells by a comprehensive analysis of PUM role and downstream targets in CRC cell tumorigenesis and by conditional Pum knockout in a mouse CRC cancer model. To test the feasibility of treating CRC by knocking down Pum 1 and Pum 2 expression, we show that intravenous injection of nanoparticles encapsulating anti-Pum 1 and Pum 2 siRNAs significantly inhibit the growth of human colorectal tumors in mice. This study reveals the biological function of PUM proteins in CRC and the underlying molecular mechanism for the oncogenic function. In addition, it provides a new approach for the treatment of CRC.

\section{Results}

\section{The expression of PUM1 and PUM2 are increased in human CRC}

To investigate the functions of PUM proteins in cancer, we analyzed two online databases including patients' clinical features and gene expression data. PUM1 showed the highest protein level in CRC compared to other cancer types and PUM2 was also highly expressed in CRC from Human Protein Atlas (www.proteinatlas.org) (Figures S1A-S1B). Analysis of Cancer Genome Atlas (TCGA) Datasets (http://software.broadinstitute.org/software/igv/tcga) revealed transcripts of Pum1 and Pum2 were higher in Hong colorectal ${ }^{26}$ and Skrzypczak colorectal ${ }^{27}$ compared with normal colon tissues (Figures S1C-S1D).

To further correlate Pum1 and Pum2 to CRC, we performed a pairwise comparison of mRNA expression in human CRC samples from 22 patients with their adjacent tissues, we found Pum 1 and Pum 2 were highly expressed in CRC clinical specimens (Fig. 1A, C). Among them, 15 patients had Pum1, 13 patients had Pum2 mRNA levels that were at least 10-fold higher than their paired adjacent tissues. Furthermore, TCGA datasets showed Pum 1 expression was positively correlated with CRC stage (Figs. 1B). The increase of

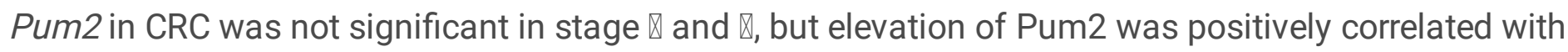
stage $₫$ and stage $\triangle$ CRC (Figs. 1D). Receiver operating characteristic (ROC) curve analysis showed that Pum 1 has higher area under the curve (AUC) values than Pum2 in CRC (Figures S1E-S1F). In addition, the expression of Pum 1 and Pum2 negatively correlated with the survival rate of CRC patients (Figure S1G, H). Together, all these data strongly correlate Pum1 and Pum2 with the progression of human CRC.

\section{Intestine-specific deletion of Pum1 and Pum2 inhibit AOM-DSS induced colon carcinogenesis in vivo}

To examine the involvement of PUM proteins in CRC, we employed a well-established azoxymethane/DSS (AOM/DSS) mouse model to evaluate the role of PUM proteins by conditional knock

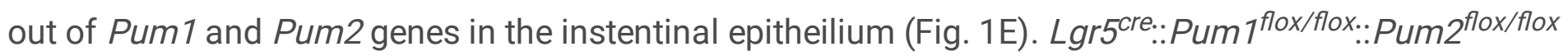

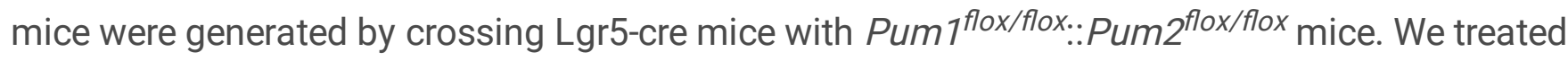

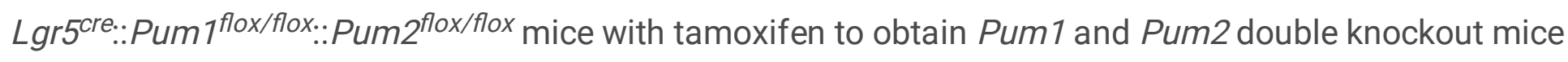




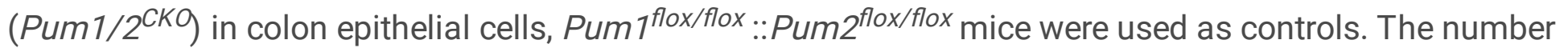
as well as the size of tumors were markedly reduced in the gut of Pum $1 / 2^{C K O}$ mice, as compared with control mice (Figs. 1F). Quantitatively, the number of tumors in Pum1/2 ${ }^{C K O}$ mice was decreased by approximately $25.9 \%, 59.4 \%$ and $91.5 \%$ with $<2 \mathrm{~mm}, 2-4 \mathrm{~mm}$ and $>4 \mathrm{~mm}$ diameter tumors respectively (Figs. 1G). Intriguingly, Pum $1 / 2^{C K O}$ mice showed longer colon length and heavier body weight than control mice (Figs. $1 \mathrm{H}$ and $1 \mathrm{I}$ ), despite that the Pum $1 / 2^{K O}$ mice are actually smaller than the wildtype controls ${ }^{28}$, suggested that control mice were more prone to tumors, since shortening of colon length in DSS-induced mice is a marker of inflammation severity, and inflammation is one of the potential pathogens of carcinogenesis in the colon ${ }^{29}$. Immunohistochemical analysis revealed no significant PUM1 and PUM2 protein in colon of Pum 1/2KO mice (Fig. 1J). Compared with the control mice, the morphology of the small intestine of knockout mice did not change, indicating that the Pum $1 / 2^{C K O}$ did not affect intestinal development (Figure S2). Surprisingly, there was a profound decrease in adenomas displaying a high grade of dysplasia in Pum $1 / 2^{C K O}$ mice (Fig. 1K), suggested the progression was inhibited. Furthermore, Ki67 and TUNEL staining revealed that Pum1/2 ${ }^{C K O}$ dramatically reduced tumor cell proliferation and slightly increased apoptosis (Figs. $1 \mathrm{~L}$ and $1 \mathrm{M}$ ). Taken together, these results demonstrate that Pum $1 / 2$ deficiency significantly inhibits initiation and development of CRC.

\section{PUM proteins contribute to CRC tumorigenicity in vivo and cell growth in vitro}

To further characterize the function of PUM1 and PUM2 in CRC, we first examined the expression of Pum 1 and Pum2 in six different CRC cell lines, with a normal human colon mucosal epithelial cell line, NCM460, as a control (Figs. 2A-2B). Pum1 was expressed in all six CRC cell lines at significantly higher levels than the normal cell line, HCT116 cells showed the highest levels of expression. Pum2 showed higher expression levels than normal cells in five out of six cancer cell lines, although the over-expression was generally not as extent as Pum1. In order to study the function of Pum 1 and Pum2 in CRC, we chose HCT116 as our cell model since it had the highest levels of Pum1 and Pum2 had expression among detected CRC cell lines. We knocked down Pum1 and/or Pum2 using siRNAs (Figures S3A), and found that Pum 1 and/or Pum 2 knockdown reduced the colony formation ability and cell-doubling time in HCT116 (Figures S3B-3E), indicating that Pum1 and/or Pum2 knockdown inhibited the growth of CRC cells in vitro. In addition, we found that Pum 1 and/or Pum 2 knockdown displayed a significantly increased proportion of cell in G0/G1 phase (Figures S3F and S3G). Moreover, knockdown of Pum1/2 did not have significant impact on the survival and migration of HCT116 cells, as indicated by the transwell assay and flow-cytometry using apoptotic markers (Figures S4). This indicates that the growth deficiency of CRC cells caused by loss of Pum was contributed by delayed G1/S transition. Notably, Pum 1 and Pum2 showed similar roles in colorectal growth, and double knockdown of Pum1 and Pum2 showed additive defects, suggesting that they play similar roles.

To remove residual protein effects and to assess whether the Pum $1 / 2$ knockdown phenotype we observed in HCT116 cells reflected a general function of Pum in CRC, we knocked out Pum 1 or Pum 2 in both HCT116 and RKO cells, using the CRISPR/Cas9 nickase method ${ }^{30}$. Our designed sgRNAs were able 
to target to all isoforms of Pum1 and Pum2, respectively (Figures S5). Immunofluorescence staining of HCT116 and RKO cells revealed a diffusely cytoplasmic distribution of both PUM1 and PUM2 (Figures S6A and S6B). These data were further confirmed by cytoplasmic nuclear fractionation, which showed that both PUM1 and PUM2 are mostly located in cytoplasmic of CRC cells (Figures S6C and S6D).

The deletion of Pum1 and Pum2 (Figs. 2C) significantly inhibited the colony formation and proliferation of HCT116 and RKO cells as indicated by two independent Pum1 (Pum1 $1^{-/-}$) and Pum2 (Pum2 ${ }^{-/-}$) knockout clones (Figs. 2D-2I). Consistently, Pum1-deficiency caused a delay in G1/S transition in both HCT116 and RKO cells (Figs. 2J-2K). In the xenograft assay, knocking out Pum 1 significantly inhibited the tumorigenicity of HCT116 and RKO cells (Figs. 2L-2Q). Pum2-/- clones displayed similar phenotype, though less severe in HCT116 cells (Figs. 2D-2Q). Together, these results indicate that both Pum 1 and Pum2 are essential to colorectal tumor tumorigenicity in vivo and cell growth in vitro.

\section{The expression of cell cycle-related genes is increased in the Pum1-deleted CRC cells}

To explore the molecular mechanism underlying the PUM function in CRC, we analyzed the transcriptome of Pum $1^{-/-}$and Pum2 $2^{-1-}$ HCT116 cells by deep sequencing (Fig. 3A, Tables S2-S3). The transcriptome of Pum $1^{-/-}$was largely affected, with 952 genes showing reduced mRNA levels and 1439 genes showing increased mRNA levels (Padj $<0.05$, Fold Change $\geq 1.5$ ). Among them, several cell cycle inhibitor genes were upregulated in Pum1 ${ }^{-/}$, including Cdkn2b, Cdkn1a/p21, Cdkn2d, Cdkn2c. The tumor suppressor function of these genes has been well-established ${ }^{31-34}$. Notably, key oncogene Myc was downregulated in Pum $1^{-/-}$HCT116 cells. Similarly, we examined the transcriptome changes in Pum2 $2^{-/-}$HCT116 cells. 1105 and 665 genes were significantly upregulated and downregulated in these cells, respectively. Cell cycle genes upregulated in Pum2 ${ }^{-/-}$were slightly different from those in $\mathrm{Pum}^{1^{-/}}$, for instance Cdkn2a, Tgfb1 were upregulated in Pum2 $2^{-/-}$cells but not $P u m 1^{-/-}$cells. Gene ontology (GO) and KEGG pathway

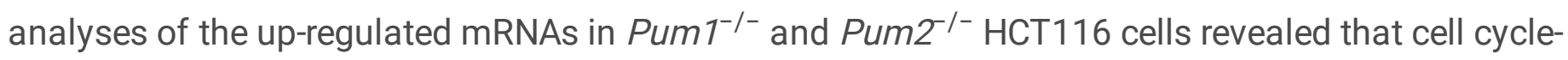
related genes were enriched in Pum inhibited genes (Figs. 3C-3F).

As PUM proteins regulate their targets largely at the post-transcriptional level, we expect a change of their targets at the protein level. Therefore, proteomics assays using TMT labeling were performed to identify protein changes between wild type, Pum1 ${ }^{-/-}$, and Pum2 ${ }^{-/-}$HCT116 cells (Fig. 3B, Tables S4-S5). Consistently, the protein level of cell cycle inhibitor gene Cdkn1a/p21 was upregulated in Pum $1^{-/-}$ HCT116 cells, suggesting that Pum 1 may repress p21 in CRC. The protein levels of Wnt signaling inhibitor Dkk1 and tumor suppressor genes Susd 2 and Pdlim5 are also upregulated in Pum2 ${ }^{-1-}$ HCT116 cells, indicating the involvement of the Wnt pathway. When GO and KEGG analyses were performed for the up-regulated proteins in Pum $1^{-/-}$and Pum2 ${ }^{-/-}$HCT116 cells, cell cycle genes were found to be enriched in both Pum $1^{-/-}$and Pum2 ${ }^{-/-}$(Figs. 3G-3J). In addition, we also performed GO and KEGG pathway analysis for down-regulated genes in Pum $1^{-/-}$and Pum2 $2^{-/-}$HCT116 cells, and found that cell cycle-related pathway was also enriched (Figures S7), but the degree of enrichment was lower than that 
in up-regulated genes. Consistent with tumorigenicity defects in Pum $1^{-/-}$and Pum2 ${ }^{-/-}$HCT116 cells, PUM proteins appears to inhibit cell cycle inhibitors to regulate CRC growth.

To determine the direct targets of PUM1, we performed PAR-CLIP experiments with Pum $1^{-/-}$HCT116 cells as negative controls, which identify binding sites at the single-nucleotide resolution on PUM1 in wild type HCT116 cells (Figures S8A and S8B). Two biological replicates were carried out for each condition and they showed large overlaps for PUM1 binding transcripts (Fig. 4A). We identified the binding sites for PUM1 using the publicly available software Paralyzer ${ }^{35}$ and found they showed the conserved TGTANATA binding motif ( $\mathrm{N}$ represents $\mathrm{A} / \mathrm{C} / \mathrm{U}$ ) (Fig. 4B). This motif was preferentially present in the $3^{\prime}$ UTRs of the mRNAs, with the second abundant region being the CDS (Fig. 4C). In addition to mRNAs, we identified the long noncoding RNA-NORAD (Figures S8C and S8D), a known target of PUM1 containing 17 binding sites ${ }^{24}$. Targets identified by PAR-CLIP were validated by qPCR (Fig. 4D and 4E). Given the observed G1/S transition delay in the Pum1-deficient cells, we mainly focused on the genes that responsible for $\mathrm{G} 1$ progression and found that an up-regulated gene in Pum1 $1^{-/-}$HCT116 cells, p21/Cdkn1a, was enriched in PUM1 PAR-CLIP and contained the canonical PRE site (TGTAnATA) presented in 3'UTR. Moreover, cell cycle and cancer pathways such as p53 signaling pathway, ErbB signaling pathway and Wnt signaling pathway are also enriched among the PUM1-direct targets (Figs. 4G and $4 \mathrm{H})$.

To explore how PUM1 regulate its targets, we further investigated 2,445 mRNAs whose expression was changed in Pum1 $1^{-/-}$HCT116 cells and contained PUM1 binding sites as indicated by the PAR-CLIP

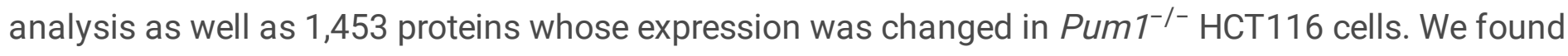
that 114 changed mRNAs and 119 changed proteins were identified as targets of PUM1 (Figs. 4I-4K, GO and KEGG analysis on these 114 mRNAs and 119 proteins, and on the target genes of PUM1 PAR-CLIP revealed that they are again enriched in cell cycle and cancer pathways, etc. (Figs. 4M-4P). The upregulation of 19 mRNAs in Pum $1^{-/-}$HCT116 cells were all validated by qRT-PCR (Fig. 4Q). These results indicate that PUM1 promotes the growth of CRC cells mainly by regulating cell cycle related genes.

\section{PUM1 directly represses $\mathrm{p} 21$ to regulate CRC growth}

Considering that p21 play an important role in G1/S phase transition and their expression is negatively correlated with survival rate of CRC (Figure S9A), we focused on the regulatory potential of PUM proteins towards p21. We evaluated p21 expression patterns, and found both the RNA and protein levels of p21 were increased in Pum1 $1^{-/-}$instead of Pum2 ${ }^{-/-}$HCT116 cells (Figs. 5A and 5B). Furthermore, p21 mRNA was significantly enriched by PUM1 and PUM2, as indicated by RIP-qPCR experiments (Figs. 5C and 5D). To tested whether PUM negatively regulate the stability of p21 mRNA, we used actinomycin to inhibit transcription and then examined the mRNA level in HCT116 cells (Fig. 5E). The stability of p21 mRNA was increased in Pum 1 $1^{-/-}$HCT116 cells, but no significant changes were found in Pum2-/- HCT116 cells. These results demonstrated PUM1 repressed $\mathrm{p} 21$ by negatively regulating its mRNA stability. We next performed dual luciferase reporter assay to evaluate the PRE contribution in PUM1-mediated p21 regulation. 3' UTR with mutated PRE resulted in a significant increase of luciferase signal over wild-type 3' 
UTR in presence of Pum1 expression construct (Figure S9B), indicating that PUM1 inhibits p21 expression via the PRE in the $3^{\prime} U T R$.

Given the possible involvement of p21 in PUM1 function, we hypothesized that deletion of PUM1 resulted reduced cell proliferation and delayed G1-S transition through directly targeting $\mathrm{p} 21$. Therefore, we mutated PRE in p21 genome by knocking in a PRE mutation donor sequence in HCT116 cells (Fig. 5F). Representative DNA sequencing results showed that we generated p21 PRE mutations ( $p 21 \mathrm{PRE}$ mut/mut) by converting TGTA to ACAT (Fig. 5G). Furthermore, PRE mutations of $p 21$ led to increased expression levels of its mRNA and protein (Fig. 5H and 5I), low affinity of its RNA to PUM1 protein (Fig. 5J), these results indicated that $p 21 \mathrm{PRE}^{\mathrm{mut} / \mathrm{mut}}$ abolished PUM1-mediated regulation on p21. We next analyzed cell growth and cell cycle of $p 21 \mathrm{PRE}^{\mathrm{mut} / \mathrm{mut}}$ cells. As previously hypothesized, $p 21 \mathrm{PRE}^{\mathrm{mut} / \mathrm{mut}}$ cells resulted in decreased colony formation, cell proliferation (Figs. $5 \mathrm{~K}-5 \mathrm{~N}$ ) and increased proportion of cells in G0/G1 phase (Fig. 50). Totally, these results demonstrated that p21 is a direct target of PUM1 and contribute to PUM1 CRC cell growth activity.

\section{PUM1 regulates its direct target genes p21 and p27 in different regulatory ways}

PUMILIO has previously been reported to facilitate miRNA binding with targeting mRNA by switching RNA secondary structure and exposing miRNA binding sites from stem-loop ${ }^{36,37}$. We assessed the effect of co-occurrence of sites of the miRNAs with PUM sites when PUM1 and PUM2 are knocked out. We classified the transcripts into five categories according to the previous methods: (i) no binding to either PUM proteins or miRNA (None); (ii) Binding only to PUM1 and/or PUM2 (PUM-only); (iii) Binding only to miRNA (miRNA-only); (iv) Binding to both a PUM protein(s) and a miRNA(s) but does not form stem-loop that span the PUM and miRNA binding sites (Both (no stem-loop)); (v) Binding to both a PUM protein(s) and a miRNA(s) and potentially form stem-loop that span the PUM and miRNA binding sites (Both (stemloop) ${ }^{38}$. Figure S10 show the comparison of the distribution of log2 fold change for these five sets of transcripts in Pum $1^{-/-}$and Pum2 $2^{-/-}$HCT116 cells. "Both (stem-loop)" and "Both (no stem-loop)" are the two groups with the most significant changes in the mRNA (Figures S10A and S10B) levels in Pum $1^{-/-}$ HCT116 cells. There was no systematic change in mRNA and protein levels in Pum2 ${ }^{-1-}$ HCT116 cells (Figures S10A and S10B). The above results indicate that miRNAs are involved in the regulation of PUM1 on target genes.

Because AGO2 is critical for miRNA-mediated regulation of target genes and AGO-PUM interaction has been reported in $C$. elegans and mammalian cells during normal development ${ }^{39}$, we investigated whether PUM1 could directly bind to AGO2 to co-regulate the expression of target genes in colorectal cancer. We used the structure of PUM1 (PDB code number: 1IB2) and AGO2 (PDB code number: 4Z4D) to predict the interaction between PUM1 and AGO2. The two combined states with the highest scores can be divided into inserting form and encircling form (Fig. 6A). A previous study showing a direct interaction between PUM2 and AGO2, in which the authors found T752 in PUM2 is critical for PUM2 interaction with AGO2 ${ }^{39}$. We aligned the PUM1 and PUM2 and found that the equivalent site in PUM1 was T874. To test the function of this residue, we conducted co-immunoprecipitation of Flag-PUM1 and Flag-PUM1(T874E) 
with AGO1 and AGO2. Flag-PUM1 interacted with both AOG1 and AGO2, and PUM1-AGO2 interaction was weakened by introducing the (T874E) mutation, but the Flag-PUM1-AG01 interaction remains unaffected (Fig. 6B). Similarly, Flag-PUM2 interaction with both AGO1 and AGO2, with PUM1-AGO2 interaction being affected by T752E mutation in PUM2 (Fig. 6C). Furthermore, the interaction between PUM1 and AGO2 was mainly through its PUF domain (Fig. 6D). This interaction appears to be in the cytoplasm where PUM1, PUM2 proteins and AGO2 co-localize (Figure S6). Considering that PUM proteins and AGO2 are RNA binding proteins, we speculate whether their binding is RNA dependent. RNase treatment abolished endogenous PUM and AGO2 interaction (Fig. 6E), which indicates that their binding is RNA-dependent. Consistent with this, the miRNA-binding deficient AGO2 (Y529E) protein ${ }^{40}$ could not bind to PUM1, while the slicer-deficient AGO2 (H634A) protein ${ }^{41}$ still bound to PUM1 (Fig. 6F). These results indicate that miRNAs may be involved in the regulation of PUM target genes.

Given the possible involvement of miRNAs in PUM1 regulation of target genes, we wondered whether miRNAs were involved in PUM1 regulation of $p 21$. We found a PRE on the 3'UTR of p21, and there are several miRNA binding sites on the 3 'UTR of p21 based on prediction ${ }^{42}$, but none of them can form a loop with PRE. Among these several miRNAs, miR-130a was reported to be highly expressed in colorectal cancer ${ }^{43}$, its binding site on the $3^{\prime}$ UTR of p21 is approximately 1000 bp away from PRE. Our colony assay and cell transfection assay on the expression of the luciferase-p21 3'UTR fusion mRNA showed that miR-130a can promote the proliferation of colorectal cancer cells, which is consistent with the function of PUM1 and PUM2 (Figures S11-S12). Hence, we chose miR-130 for further study.

We then investigated the regulation of miR-130a on p21. Western blotting analysis revealed that overexpression of miR-130a reduced p21 protein level in both WT and Pum $1^{-/-}$HCT116 cells (Figs. $6 \mathrm{H}$ and S12A-S12C). However, anti-miR-130a only increased p21 protein level in Pum1 ${ }^{-/-}$HCT116 cells, but not in WT HCT116 cells (Figs. 6I and S12A-S12C). These results indicate that PUM1 is the dominant regulator of p21 in HCT116 cells compared with mir-130a. Moreover, miR-130a inhibited the expression of p21 by directly binding to its 3'UTR (Figures S12D-S12E). Meanwhile, the regulation of PUM1 and miR130a on p21 was relatively independent (Figure S12F). For p27, there are two PRE motifs and two miR$221 / 222$ binding sites in its $3^{\prime} U T R$. The distance between one PRE and one miR-221/222 binding site is only 12 bp, which could form a loop structure and thus p27 belongs to "Both (stem-loop)" (Fig. 6J) ${ }^{38}$. While the effect of overexpression or inhibition of miR-222 on p27 in Pum 1 ${ }^{-/-}$HCT116 cells was smaller than that of miR-130a on p21 (Fig. 6K and L). The above results indicate the possibility that, without PUM1 binding, the loop composed of PRE and miR-222 binding sites was not be opened. This prevented the binding sites of miR-222 to be exposed for miR-222 binding, so that miR-222 could only regulate $\mathrm{p} 27$ expression by binding to another binding site in the p27 3 'UTR.

We verified western blotting results by luciferase reporter assay in WT and Pum $1^{-/-}$HCT116 cells. Transfecting miR-130a mimics reduced luciferase activity of p21-3'UTR-WT or p21-3'UTR-PRE-MUT in both WT and Pum1 ${ }^{-/-}$HCT116 cells (Figs. 6M-6N). As for the regulation of miR-222 on p27-3'UTR luciferase activity, we found that transfecting miR-222 mimics inhibited luciferase activity of p27-3'UTR- 
WT in WT and Pum 1 $1^{-/-}$HCT116 cells (Fig. 60). To examine the regulatory effect of the two miR-222 binding sites in p27-3'UTR, we mutated first miR-222 binding site of the $3^{\prime} U T R$, resulting in a mutant reporting construct p27-3'UTR-miR-222-MUT1. Transfection of miR-222 mimics only reduced luciferase activity of p27-3'UTR-miR-222-MUT1 in WT HCT116 cells but not in Pum 1 ${ }^{-/-}$HCT116 cells (Fig. 6P). This indicates the possibility that, without PUM1 in Pum1 $1^{-/-}$HCT116 cells, the loop formed by PRE and miR222 binding site cannot be opened for miR-222 to bind to the second binding site - the only other miR222 binding site in p27-3'UTR-miR-222-MUT1 (Fig. 6P) ${ }^{36}$. In support of this notion, when the first PRE was mutated (p27-3'UTR-PRE-MUT1), overexpression of miR-222 reduced luciferase activity in both WT and Pum $1^{-/-}$HCT116 cells (Fig. 6Q). This indicates that the loop formed by PRE and miR-222 binding site was disrupted, opening up the second miR-222 binding site, regardless of the presence or absence of PUM1 ${ }^{36}$. As a negative control, when the second PRE, not expected to form a stem-loop with a miR-222 binding site, was mutated (p27-3'UTR-PRE-MUT2), the regulatory effect of miR-222 on p27-3'UTR-PREMUT2 is similar to that of p27-3'UTR-WT (Fig. 6R), indicating that the mutation of the second PRE site does not affect the binding of miR-222 to its binding site. A previous study demonstrated that PUM1 binding induces a local change in RNA structure of p27 that favors association with miR-221 and miR222, efficient suppression of p27 expression ${ }^{36}$. However, they found that only the binding site of miR$221 / 222$ that formed loops with PRE played a role of miR-221/222 in inhibiting p27. Our findings indicate that the first miR-221/222 binding site also plays a role in the inhibition of p27 (Figs. 60 and 6P). These findings indicate that PUM1 promotes the proliferation of colorectal cancer cells partly by regulating p21 and p27 in different regulatory ways: For PUM1 regulation of p21, is a miRNA independent manner. For $\mathrm{p} 27$, requires coordinated regulation by $\mathrm{miR}-221 / 222$.

\section{Nanoparticleencapsulated PUM siRNA partially inhibits the development of CRC}

To explore the possibility of using PUM1 and PUM2 as targets for anti-cancer treatment, we further investigated the in vivo antitumor activity of siRNA-loaded nanoparticles using the orthotopic colon cancer models (Figs. 7A) ${ }^{44}$. Two colorectal tumor models, HCT116-luc and COLO205-luc, were used in this study. The established polyethylenimine (PEI)-coated, glutathione (GSH)-responsive, mesoporous silica nanoparticles (MSN) were adopted for anti-Pum 1 and Pum2 siRNA delivery ${ }^{44}$. Empty MSN without PEl coating were spherical with a diameter of $\sim 90 \mathrm{~nm}$ as shown in transmission electron microscopy (TEM) (Figure S13A). The hydrodynamic diameter of empty MSN without PEl determined by DLS were $100 \mathrm{~nm}$ (Figure S13B), a little larger than the dimension acquired in TEM. PEl coating and siRNA loading slightly increased the nanoparticle size. The zeta potential reversed from negative $(-33 \mathrm{mV})$ for empty MSN to highly positive (34 mV) for PEl-coated siRNA-loaded MSN (Figure S13C). siRNA molecules could be efficiently released in the presence of $5 \mathrm{mM} \mathrm{GSH}$-contained PBS, which well mimicked the environment of cancer cell cytoplasm (Figure S13D) ${ }^{45,46}$. Tumor targeting of the siRNA-loaded nanoparticles was observed using IVIS Spectrum CT multimodal imaging system (Fig. 7B). The Cy5 labeled siRNA@MSN specifically targeted luciferase-labeled tumor cells $24 \mathrm{~h}$ after tail vein injection, demonstrating the feasibility of our approach. 
We transplanted HCT116 and COLO205 tumor cells subcutaneously into four-week-old male BALB/c nude mice. 28 days after the implantations, mice were divided into five groups ( 5 mice per group) according to the comparable bioluminescence intensity of the tumors and intravenously injected with various nanoparticle formulations every 3 days with four repetitions (day 0, 3, 6, 9; Fig. 7C).

Bioluminescence imaging was used to monitor tumor growth every week (day 7, 14, 21, 28; Figs. 7D and 7H). For the HCT116 tumor model, non-specific siRNA control (siNC@MSN) did not delay the tumor growth. In contrast, injection of nanoparticles loaded with anti-Pum1 siRNA (siPum1@MSN) or anti-Pum2 siRNA (siPum2@MSN) exhibited dramatical effect of inhibiting tumor growth. Particularly, in the mice treated with both anti-Pum1 and anti-Pum2 siRNAs (siPum1/2@MSN) showed the strongest antitumor effect (Fig. 7E). At the end of the experiment (day 30), the mice were sacrificed and tumors were excised for weighting. The tumor weights of mice treated with siPum1/2@MSN were 1.6-3.5 fold lighter than those treated with other controls (Fig. 7F). During the treatment, mice body weight from all groups had a similar increase, indicating no overt toxicity (Fig. 7G).

Because metastasis is the main cause of death in patients with CRC ${ }^{47}$, with nearly $60 \%$ of CRC patients have metastasis in the liver and up to $35 \%$ of the metastasis only occurs in this organ ${ }^{48}$, we also examined the anti-metastatic effect of the treatment. On day 30, major organs (heart, liver, spleen, lung, and kidney) from all the mice were excised for anti-metastasis evaluation using bioluminescence imaging (Figure S14). In the saline control group, all the mice had metastases in the liver. 2 of the five mice had spleen and lung metastases. No metastases were observed in kidney. The mice in the siNC@MSN control group had similar metastasis pattern to that of the saline group. In contrast, metastases in liver, spleen, and lung were significantly inhibited with the treatment of siPum1@MSN or siPum2@MSN. No metastases in spleen for siPum1@MSN group and in lung for siPum2@MSN group were obtained. For the treatment with siPum1,2@MSN, there were no metastases in all detected organs, indicating the best anti-metastasis effect. Similar antitumor effect was also observed in the COLO205 tumor-bearing mice model (Figs. 7I-7K and S15). Taken together, Pum1 or/and Pum2 siRNA delivery by nanoparticles can prevent further growth of colorectal tumors in vivo, and Pum1/2 are expected to be potential targets for the treatment of CRC.

\section{Discussion}

In this paper, we reported the requirement of PUM1 and PUM2, two members of the human PUF protein family, for the initiation and progression of CRC. Furthermore, we discovered that such a cancer cell growth-promoting function was in part achieved by inhibiting p21, a negative regulator of cell cycle. Finally, we showed that treating CRC models by intravenous injection of nanoparticle-packaged anti-PUMI siRNAs effectively reduced tumor growth. These findings provide new insights into molecular mechanism that contributes CRC as well as a potentially new approach for treating CRC.

\section{Different functions of PUM proteins in diverse tumors}


In recent years, a number of studies have reported that human PUM proteins play an important oncogenic role. However, the function of PUM proteins in different tumors is different, and there is a debate on whether PUM proteins are oncogenes or tumor suppressors. Kedde et al. reported that PUM1 co-regulate the expression of the cell cycle inhibitory protein p27 with classical oncogenic miRNAs miR-221 and miR222 in human breast cancer cells and HEK293 cells ${ }^{36}$. Because the knockdown of PUM1 reduced the number of cells in S phase and inhibit cell proliferation, the authors suggested that PUM1 may promote tumorigenesis in breast cancer. In non-small cell lung cancer, tumor suppressor miR-340 directly binds to the 3'UTR of Pum 1 and Pum 2 mRNA and inhibits their expression, thus reducing PUM1 and PUM2 that are required for the miR-221/222 interaction with the $p 273^{\prime} U T R{ }^{49}$. In addition, recent studies in leukemia cells have shown that PUM proteins can directly bind to the mRNA of the transcription factor FOXP1 to increase the expression level of FOXP1 protein, thereby increasing the proliferation of hematopoietic stem cells and myeloid leukemia cells ${ }^{50}$. Moreover, PUM1 and PUM2 inhibit kinase activator GC-32 by deadenylation to promote the growth of EB virus immortalized B cells ${ }^{51}$. Furthermore, PUM1 can promote the development of ovarian cancer ${ }^{52}$. All these studies are similar to our finding that knockout or knockdown of PUM1 and PUM2 blocked cell G1/S phase transition by upregulating p21 (Figs. 2 and 5) and significantly inhibited the proliferation of CRC cells. All of the above studies have revealed the requirement of PUM proteins for oncogenesis.

However, Miles et al. found that PUM1 and PUM2 co-inhibit the expression of oncogene E2F3 with miRNA in bladder cancer ${ }^{25}$. In this cancer, even though the level of PUM1 or PUM2 protein itself did not change significantly in tumor cells, the level of the miRNAs (miR-503, miR125b) synergizing with PUMILIO proteins was decreased significantly, which allowed oncogenesis. In triple-negative breast cancer cells, PREs in the 3'UTR of oncogene c-jun were deleted due to alternative polyadenylation, which promotes the cancer. This also indicates an oncosuppressor role of PUM proteins ${ }^{53}$. Thus, PUM proteins have opposite functions in different cancers. This difference may be due to their targeting of different genes or partnering with different proteins in different tumors.

\section{PUM1 directly represses p21 in human colorectal cancer}

A worth-noting finding of our study is that $\mathrm{p} 21$ is a direct target of PUM1. Previously study reported that PUM1/2 positively regulated FOXP1, and FOXP1 mediated PUM growth-promoting activities by repressing expression of p21 in human hematopoietic stem /progenitor cells and leukemic cells ${ }^{50}$. Their results suggested that PUM1 negatively regulated p21, which was consistent with our finding. However, we provide strong evidence that PUM1 directly bound and repressed p21 via 3'UTR PRE instead of through FOXP1 or other factors. Our results showed that PUM1 significantly down-regulated both mRNA and protein levels of p21 by promoting p21 mRNA turnover, possibly through the well-known pathway in which PUM proteins interact with the Ccr4-Pop2-NOT deadenylase complex to inhibit mRNA stability. This eventually resulting colorectal cancer proliferation and shortened G1/S transition.

Based on our results, we propose the following model to illustrate the function of PUM proteins in human CRC: PUM1 and PUM2 are highly expressed in CRC and bind to $p 21 \mathrm{mRNA}$ to reduce its expression. This 
relieves p21 suppression of cell cycle and cell growth mechanisms, allowing CRC progression. Either Pum 1 deletion or $p 21$ RPE mutation abolish PUM repression of $p 21$, which results in CRC growth defects and delayed G1/S transition.

\section{Whether PUMILIO, as a widely expressed protein in cells, can be a therapeutic target for CRC}

PUM proteins are widely expressed in many tissues $19,54,55$, so if PUM proteins are used as targets for cancer treatment, a priori one needs to determine whether such a treatment will cause broad side effect. Remarkably, Pum1;Pum2 double knockout in small intestinal epithelial cells did not cause detectable effect on intestinal homeostasis and function in our mouse colorectal tumor model (AOM/DSS; Figure S2). Instead, we found that conditional knockout of Pumilio effectively blocked the occurrence and development of colorectal tumors (Figs. 1E-1I). Moreover, when we treated colon orthotopic implant tumors by tail vein injection of nanoparticles encapsulated with anti-Pumilio siRNAs, these siRNAs prevented the further growth of colorectal tumors without obvious effects on other organs (Figs. 6 and S16). This might be because PUM proteins are expressed in colorectal tumors at higher levels than in normal tissues, and also possibly because nanoparticles have a tendency to accumulate in tumor cells. Our results point to the feasibility of using PUM proteins as targets for CRC treatment. A systematic examination of the biodistribution of Pum $1 / 2$ loaded nanoparticles and the levels of Pum 1 and Pum2 in other tissues in the future will further test this feasibility.

\section{Materials And Methods}

\section{Mice}

Male or female BALB/c nude mice were purchased from Shanghai Lingchang Biotechnology Co., Ltd (Shanghai, China). All animals were housed and maintained in pathogen-free conditions. All animal experiments were performed in compliance with the Guide for the Care and Use of Laboratory Animals and approved by the Institutional Biomedical Research Ethics Committee of the ShanghaiTech University or Shanghai Jiao Tong University School of Medicine (SJTU-SM).

\section{Cell Culture}

HCT116, RKO, COLO205, LOVO, SW480, SW620, HT29 cells were cultured according to the culture methods of ATCC.

\section{Cell Cycle}

Cells within $2-5 \times 10^{6}$ were collected, then wash the cells twice with $1 \mathrm{ml}$ cold PBS, each time centrifuged at $400 \mathrm{~g}$ for 5 minutes at $4^{\circ} \mathrm{C}$. Discard most of the supernatant and gently bounce the cell sediment evenly, so that the volume of cell suspension is about 50-100ul. Use a whirlpool oscillator to keep the cells in suspension, slowly drop by drop add $1 \mathrm{ml}$ of ice-cold $75 \%$ ethanol (pre-cooled at $-20^{\circ} \mathrm{C}$ ). Fixed overnight at $4{ }^{\circ} \mathrm{C}$ or for 4 hours at $-20^{\circ} \mathrm{C}$. Cells were collected by centrifugation at $1000 \mathrm{~g}$ for 5 min at $4{ }^{\circ} \mathrm{C}$ before analysis. Wash cells once with $1 \mathrm{ml}$ cold PBS, resuspend cells with $400 \mu \mathrm{l}$ cold PBS. Add $400 \mu \mathrm{l} \mathrm{PI}$ 
Staining Solution/RNase solution, gently mix and incubate for 30-60 minutes in the dark at $4^{\circ} \mathrm{C}$ then analyzed by flow cytometry. The maximum excitation wavelength is $488 \mathrm{~nm}$.

\section{Cell Apoptosis}

HCT116 or RKO cells were stained with the FITC Annexin V apoptosis detection kit (556547, BD Biosciences) or PE Annexin V apoptosis detection Kit (559763, BD Biosciences) according to the manufacturer protocol and analyzed early- and late-stage apoptosis by FACS (FACS AriaTM IIII, BD Biosciences).

\section{Cell Growth Curve and Colony Formation}

For growth curve assay, a total of $1 \times 10^{4} \mathrm{HCT} 116$ or RKO cells per well were seeded in 12-well plate, triplicate wells were seeded. Count cells every 24 or 48 hours for a total of 5-8 days. For colony formation assay, a total of 1000 HCT116 or RKO cells per well were seeded in 6-well plate, triplicate wells were seeded. After 10 days, cells were stained with Crystal Violet Staining Solution (C0121, Beyotime).

\section{RNA Extraction and Real-time PCR}

RNA extraction and real-time PCR analysis were performed according to previous protocol ${ }^{56}$. The mRNA and miRNA levels were assessed by SYBR Green-based quantitative real-time PCR with gene-specific primers (Supplementary Table S1).

\section{Western Blot}

Western blot analysis was performed according to our previous method ${ }^{57}$. The following primary antibodies were used: PUM1 (ab92545), PUM2 (ab92930), AG01(ab5070), AG02 (ab57113), Lamin A/C (ab108595) were purchased from Abcam. p21 (2947S), p27 (3686S), Tubulin (2125S), GAPDH (2118S) were purchased from Cell Signaling Technology.

\section{RNA Immunoprecipitation (RIP)}

After the cells grow to $90 \%$ density in a $10 \mathrm{~cm}$ dish, the medium is removed, wash twice with PBS, add 1 $\mathrm{ml}$ of cell lysate and lyse on ice for $30 \mathrm{~min}$. Cells and lysate were collected with a cell scraper and centrifuged in $1.5 \mathrm{ml}$ centrifuge tube at $120000 \mathrm{rpm}$ for $15 \mathrm{~min}$. Take the supernatant, reserve $50 \mu \mathrm{l}$ as input, and divide the cell lysate into two parts. Add $2 \mu \mathrm{g}$ PUMILIO antibody (Abcam) and $2 \mu \mathrm{g} \lg$, respectively, and incubate overnight at $4^{\circ} \mathrm{C}$. Protein $\mathrm{A}$ magnetic beads were washed twice with cell lysate, and $50 \mu$ of the washed magnetic beads were added to $1.5 \mathrm{ml}$ centrifugal tube incubated overnight, and incubated at $4^{\circ} \mathrm{C}$ for 4 hours. The incubated magnetic beads were washed five times with a magnetic frame and NT2 buffer for 5 min each time. Remove supernatant, collect magnetic beads, add TRIzol reagent (Invitrogen) and extract RNA.

\section{Photoactivatable Ribonucleoside-Enhanced Crosslinking and Immunoprecipitation (PAR-CLIP)}

PAR-CLIP was performed following the previous protocol ${ }^{18}$. For each condition (namely, WT1-Pum1-PARCLIP, WT2-Pum1-PAR-CLIP, K01-Pum1-PAR-CLIP, KO2-Pum1-PAR-CLIP), we grew $15 \mathrm{~cm}$ cell culture plates

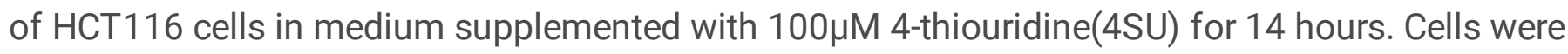


washed once with cold PBS and then cross-linked on ice using $0.15 \mathrm{~J} / \mathrm{cm}^{2}$ of $365-\mathrm{nm}$ ultraviolet light in a Stratalinker. Cells were then scraped from culture dishes, transferred into a 15-ml Falcon tube, washed once with PBS, pelleted by centrifugation at $500 \mathrm{~g}$ for 5 minutes and flash-frozen in liquid nitrogen for storage at $-80^{\circ} \mathrm{C}$.

On the day of the experiment, cell pellets were thawed on ice, suspended in 3 volumes (the volumes of cell pellets were about $2 \mathrm{ml}$, hence $6 \mathrm{ml}$ of lysis buffer were added) of lysis buffer $(20 \mathrm{mM}$ Tris- $\mathrm{HCl} \mathrm{pH}=$ 7.4, $150 \mathrm{mM} \mathrm{NaCl}, 1 \mathrm{mM}$ EDTA, 1\% (V/V) IGEPAL $®$ CA-630, 0.5 mM DTT, cOmplete ${ }^{\text {TM }}$ EDTA-free Protease Inhibitor Cocktail Tablets, and PhosStop Tablets) and incubated on ice for 30 minutes. Cell lysate was collected by centrifugation at $13,000 \mathrm{~g}$ at $4^{\circ} \mathrm{C}$ for 30 minutes and further filtered by $0.45 \mu \mathrm{M}$ pore size membrane filter (Millipore, Cat\#: HAWP04700). Partial RNA digestion was performed before immunoprecipitation with $1 \mathrm{U} / \mu \mathrm{l}$ RNase T1 (Thermo Fisher Scientific, EN0541) at $22^{\circ} \mathrm{C}$ for 15 minutes. In the meantime, the Protein A beads-antibody complexes were prepared, for each sample, $0.25 \mathrm{mg} / \mathrm{ml}$ Pum1 antibody (Abcam, Cat\#: ab92545) was conjugated to $100 \mu \mathrm{l}$ protein A beads at $4{ }^{\circ} \mathrm{C}$ for 2 hours, unbound antibody was removed and added the pre-cleared lysates to antibody-coupled beads and incubated overnight at $4^{\circ} \mathrm{C}$.

The following day, wash the beads five times with wash buffer $[20 \mathrm{mM}$ Tris- $\mathrm{HCl} \mathrm{pH}=7.4,200 \mathrm{mM} \mathrm{NaCl}$, $0.05 \%$ (v/v) IGEPAL $®$ CA-630, 0.5 mM DTT, cOmpleteTM EDTA-free Protease Inhibitor Cocktail Tablets, and PhosStop Tablets) and digest with $10 \mathrm{U} / \mu \mathrm{l}$ RNase $\mathrm{T} 1$ at $22^{\circ} \mathrm{C}$ for 15 minutes, then wash the beads three times in high salt wash buffer $\left(20 \mathrm{mM}\right.$ Tris- $\mathrm{HCl} \mathrm{pH}=7.4,300 \mathrm{mM} \mathrm{NaCl}, 0.05 \%$ (v/v) IGEPAL ${ }^{\circledR}$ CA630, 0.5 mM DTT, cOmpleteTM EDTA-free Protease Inhibitor Cocktail Tablets, and PhosStop Tablets). Immunopurified protein-RNA complexes were dephosphorylated by resuspending beads in $200 \mu \mathrm{l}$ dephosphorylation mix ( $1 \times$ NEBuffer 3 (New England Biolabs, Cat\#: B7003S), $0.5 \mathrm{U} / \mu \mathrm{l}$ calf intestinal alkaline phosphatase (Thermo Fisher Scientific, Catalog \# M0290)) and incubated at $37^{\circ} \mathrm{C}$ for 10 minutes. Then, wash the beads two times with $1 \times$ NEBuffer 3 and two times in PNK buffer without DTT (50 mM Tris- $\mathrm{HCl} \mathrm{pH}=7.4,50 \mathrm{mM} \mathrm{NaCl}, 10 \mathrm{mM} \mathrm{MgCl} 2 \cdot \mathrm{H}_{2} \mathrm{O}$ ). Next, 3 ' ligation was performed according to the NEBNext Multiplex Small RNA Library Prep Set for Illumina (Set 1) (New England Biolabs, Cat\#: E7300S).

The next day, wash the beads two times with high salt wash buffer followed by two times with PNK buffer without DTT. Then the protein-RNA complexes were phosphorylated by resuspending beads in $100 \mu$ phosphorylation mix ( 1 × T4 DNA ligase buffer (Thermo Fisher Scientific, Cat\#: B69), 1 U/ $\mu \mathrm{l} \mathrm{T4}$ Polynucleotide kinase (Thermo Fisher Scientific, Cat\#: EK0031)) and incubated at $37^{\circ} \mathrm{C}$ for 30 minutes. Then wash the beads three times with wash buffer. Subsequently, NuPAGE LDS Sample Buffer (Thermo Fisher Scientific, Cat\#: NP0007) was added to each sample and incubated at $95^{\circ} \mathrm{C}$ for 5 minutes. Protein-RNA complexes were resolved using NuPAGE 4-12\% Bis-Tris-HCl Gels (Thermo Fisher Scientific, Cat\#: NP0335BOX) and desired complexes were excised from gel using a clean scalpel. The gel pieces were transferred to a D-tube Dialyzer Midi tube (Millipore, Cat\#: 71506) and $800 \mu \mathrm{l} 1 \times$ SDS buffer (Thermo Fisher Scientific, Cat\#: NP0001) was added. After electroeluted the cross-linked RNA-RBP complex, transferred the solution to tubes, added $800 \mu$ proteinase $\mathrm{K}$ buffer $(100 \mathrm{mM}$ Tris- $\mathrm{HCl} \mathrm{pH}=7.4$, 
$150 \mathrm{mM} \mathrm{NaCl}, 12.5 \mathrm{mM}$ EDTA, 2\% (V/V) SDS (Bio-Rad, Cat\#:1610418)) with 1.2mg/ml proteinase K (New England Biolabs, Cat\#P8107S) and incubated at $55^{\circ} \mathrm{C}$ for 30 minutes. Then recover the RNA with Trizol reagent according to the manufactory's protocol. The following steps of library preparation were performed as described according to the NEBNext Multiplex Small RNA Library Prep Set for Illumina (Set 1).

\section{Analysis of PAR-CLIP data}

The forward read1 was taken from the paired-end sequenced PAR-CLIP data of each sample. This is because the RBP (RNA-binding protein)-bound fragments are short (<70bp), the $150 \mathrm{bp}$ long paired-end read1 and read2 will share the same fragment, thus one read (either read1 or read2) is enough. First, TrimGalore (version 0.4.4_dev, http://www.bioinformatics.babraham.ac.uk/projects/trim_galore/) was used to trim off the adapter sequences and only reads in the size range of 17-50 nt were retained for downstream analyses. Secondly, the size-selected reads were aligned to the human hg19 genome using 0,1 and 2 mismatches sequentially using bowtie (version 1.2.1.1) ${ }^{58}$ (sequential procedure: 1 ) map reads to the genome using 0 mismatch (-v 0$)$, retain the mapped reads, and align the unmapped reads to the genome using 1 mismatch $(-v 1) ; 2)$ retain the 1-mismatched reads and map the unmapped reads to the genome using 2 mismatches ( $-v$ 2)). A maximum of two mismatches were used as the RBP-binding sites will show up to T-to-C conversions during the treatment step of the PAR-CLIP protocol. To increase the signal-to-noise ratio, only reads resulting from up to $2 \mathrm{~T}$-to-C conversions were selected, i.e., reads from other mismatches were discarded. Customized scripts and commands (available upon request) were developed to process aligned SAM files to identify reads that perfectly aligned to the genome, and those aligned to the genome with 1 or 2 mismatches resulting from T-to-C conversion(s) only. Subsequently, the three SAM files ( 0 mismatch, 1 T-C converted SAM, 2 T-C converted SAM) were merged and sorted using

samtools (version 1.4.1) ${ }^{59}$, and acted as the input for PARalyzer (v1.5) ${ }^{60}$. The parameters in the ".ini" file used as the input for PARazyler are as follows:

BANDWIDTH = 3,

CONVERSION $=\mathrm{T}>\mathrm{C}$,

MINIMUM_READ_COUNT_PER_GROUP = 5, MINIMUM_READ_COUNT_PER_CLUSTER $=2$, MINIMUM_READ_COUNT_FOR_KDE $=3$,

MINIMUM_CLUSTER_SIZE $=11$, MINIMUM_CONVERSION_LOCATIONS_FOR_CLUSTER $=2$, MINIMUM_CONVERSION_COUNT_FOR_CLUSTER $=2$, MINIMUM_READ_COUNT_FOR_CLUSTER_INCLUSION = 1, MINIMUM_READ_LENGTH = 20, MAXIMUM_NUMBER_OF_NON_CONVERSION_MISMATCHES = 1,

GENOME_2BIT_FILE = Homo_sapiens.GRCh19.dna.primary_assembly.2bit.

The bound regions identified by PARalyzer from the "resulting.cluster.file" were processed and intersected with the hg19 gene annotations using bedtools ${ }^{61}$ for detailed annotation (for instance, $3^{\prime} U T R$, or CDS, 
$5^{\prime} \mathrm{UTR}$, or intron of particular genes of the hg19 genome). Two replicates for each genotype (wild type and knockout) were analyzed separately. To identify the PUM1-bound genes, the bound genes from each replicates of the wild type were combined (take the union not the intersect), and those genes identified to be bound by PUM1 in the knockout were excluded, as they presumably contain non-specific binding sites (not resulting from PUM1 but other non-specific regions of the antibody). The bound regions were analyzed for enriched motif analysis and visualized in IGV ${ }^{62}$ to infer the potential binding affinity (percentage of T-to-C conversions among all mapped reads covering the region of interest).

\section{Mass Spectrometry and RNA Deep Sequencing}

Mass spectrometry was performed with the TMT kit from Thermo Fisher (\#90110) and followed the product instructions. The selection criteria for up-regulated proteins were that the number of unique peptides was more than 1 and the up-regulated fold was higher than 1.2. For RNA sequencing, wild type, Pum $1^{-/-}$and Pum2 $2^{-1-}$ HCT116 cells were collected. Total RNA was isolated using TRIzol (Invitrogen, 15596026) according to the protocol of the manufacturer. mRNAs and small RNAs were sequenced with an Illumina HiSeq3000 platform (Lie Bing Co., Shanghai, China). The procedure on analyses of RNA-Seq data followed the same as described in Shi et al. 2020. Briefly, paired-end reads were mapped onto the hg38 reference using STAR, HTSeq-count software was used to quantify the reads mapped to each gene. The R package DESeq2 was used to identify significantly differentially expressed genes in uum $^{-1 /-}$ / Pum2 $2^{-/}$compared with WILD TYPE HCT116 cells (adjusted $p$-value $\leq 0.05$ or fold change $\geq 1.5$ ).

\section{Immunofluorescence Microscopy}

Immunofluorescence microscopy assay was performed according to a previously described standard method ${ }^{57}$.

\section{Immunohistochemistry}

Formalin-fixed, paraffin-embedded colorectal tumor tissue blocks from mice were used in our investigation. Immunohistochemical staining was performed using primary antibodies and HRPconjugated secondary antibodies. The antibodies used include PUM1 (ab92545, Abcam), PUM2 (ab92930, Abcam), p21 (2947S, Cell Signaling Technology), p27 (3686S, Cell Signaling Technology) and anti-Ki67 (sc-7846, Santa Cruz Biotechnology Inc). TUNEL staining was performed using an in situ Cell Death Detection Kit (Roche) based on the manufacturer's instructions.

\section{Luciferase Reporter Assay}

The 3'UTRs of p21, p27 were amplified and cloned into psiCHECK2 vector (C8021, Promega) using primers specified in Supplementary Table S1. In the 3'UTR reporter assay, HCT116 cells in 24-well plates were transfected with $100 \mathrm{ng}$ of the psiCHECK2-p21-3'UTR-WT or psiCHECK2-p27-3' UTR-WT or 3'UTR mutant plasmids with mutations in PUM1, miR-130a or miR-221/222 binding sites and $100 \mathrm{nM}$ mimics or $100 \mathrm{nM}$ antisense (RiboBio) or $500 \mathrm{ng}$ pGL3-PUM1 plasmid along with $2 \mu$ Lipofectamine 2000 (Invitrogen). Lysates were harvested after 48 hours of transfection. The reporter activity was measured with the Dual Luciferase Assay (E1910, Promega).

\section{Tumorigenesis Assay}


Four-week-old BALB/c nude mice (male) were purchased from Shanghai Lingchang Biotechnology Co., Ltd (Shanghai, China). All experiments were performed under the guidelines for the care and use of laboratory animals. BALB/c nude mice were randomly divided into two groups ( $\mathrm{n}=8$ per group). Then, wild type, Pum 1 ${ }^{-/-}$and Pum2 ${ }^{-/-}$HCT116 $\left(2 \times 10^{6}\right)$ or RKO $\left(3 \times 10^{6}\right)$ cells in $0.1 \mathrm{ml}$ of medium mixture (medium: matrigel $=1: 1$ ) were subcutaneously injected into the nude mice. The tumor size was measured at time intervals as indicated in Figs. $2 \mathrm{M}$ and 2P. The mice were euthanized by cervical dislocation and the tumors were dissected. The tumor weight was measured, and the tumor volume was calculated using the following formula: $D \times d^{2} / 2$, with ' $D$ ' representing the longest diameter and ' $d$ ' representing the shortest diameter.

\section{AOM/DSS Model}

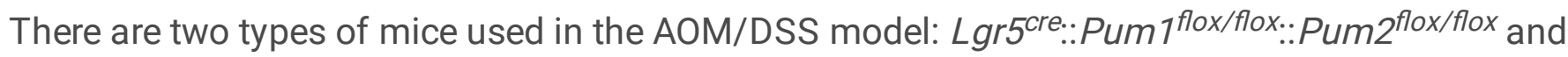

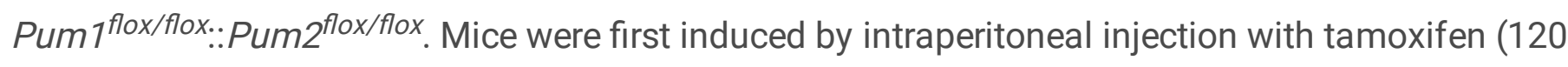
$\mathrm{mg} / \mathrm{kg}$ ) every other day for a total of 7 days. Sixteen days later, mice were injected intraperitoneally with $12.5 \mathrm{mg} / \mathrm{kg}$ azoxymethane (Sigma, St.Louis, MO). Dextran sodium sulfate (DSS) $2.5 \%$ (wt/vol) (molecular mass, 36-50 kilodaltons; Meilun biotechnology) was given for 5 days. Mice were then given regular drinking water for 14 days, followed by 1 additional cycle with DSS. In the third cycle, the DSS was adjusted from $2.5-2 \%$. On day 65 , mice were sacrificed for testing.

\section{Preparation and Characterization of siRNA-loaded MSN}

We previously developed a woven PEI (1.8 kDa)-coated, glutathione (GSH)-responsive, mesoporous silica nanoparticle (MSN) for nucleic acid (miRNA-145) delivery ${ }^{44}$. The nanoparticles can quickly escape from the lysosome into the cytosol for miRNA-145 delivery, mediated by the proton sponge effect of PEI ${ }^{58}$. The reducing environment (0.5-10 mM GSH) ${ }^{45,46}$ within tumor cells removed the PEI from the MSN surface through hydrolyzing the disulfide bond and thus allow nucleic acid release in the cytoplasm ${ }^{44}$. Here, this smart nanocarrier was adopted for the delivery of siPum1 and 2, which were complexed with MSN to generate siPum1, 2@MSN. Transmission electron microscopy (TEM) images were acquired on a FEI Talos F200X system. The hydrodynamic size and zeta potential of nanoparticles were determined through dynamic light scattering (DLS) and measured using a ZetaSizer Nano ZS instrument (Malvern, Worcestershire, UK).

siRNA (FAM labeled) release test was performed in the presence of $5 \mathrm{mM}$ GSH-contained PBS in the centrifuge tubes. The tubes were placed in gas bath at $37^{\circ} \mathrm{C}$ shaking at $100 \mathrm{rpm}$. At pre-determined time point $(1,2,4,8,12,24 \mathrm{~h})$, empty DPPC liposomes $(-30 \mathrm{mV}, 120 \mathrm{~nm}, 25 \mathrm{mg} / \mathrm{ml})$ was added to bind free PEI and PEI-siRNA complex. Then, the solution was transferred into Ultra Centrifugal Filters (molecular weight cut-off $50 \mathrm{kDa}$ ), and then centrifuged under $5000 \mathrm{rpm}$ at $4^{\circ} \mathrm{C}$ for $30 \mathrm{~min}$. FAM-labeled siRNA (Ex $488 \mathrm{~nm}$, $\mathrm{Em} 520 \mathrm{~nm}$ ) in the filtrate was collected for the quantification of nucleic acid release. The in vivo tumor targeting of the siRNA-loaded nanoparticles $24 \mathrm{~h}$ after i.v. injection was examined using the IVIS Spectrum/CT imaging system (PerkinElmer, USA) as previously described ${ }^{44}$. 
siRNA molecules specifically targeting the mRNA of Pum1 and Pum2 were purchased from RIBOBIO (China). siPum1: GGTCAGAGTTTCCATGTGA, siPum2: CTGAAGTAGTTGAGCGCTT ${ }^{24}$. siRNA (FAM labeled) release test was performed in the presence of $5 \mathrm{mM} \mathrm{GSH}$-contained PBS in the centrifuge tubes. The tubes were placed in gas bath at $37^{\circ} \mathrm{C}$ shaking at $100 \mathrm{rpm}$. At pre-determined time point $(1,2,4,8,12$, $24 \mathrm{~h})$, empty DPPC liposomes $(-30 \mathrm{~nm}, 120 \mathrm{~nm}, 25 \mathrm{mg} / \mathrm{ml})$ was added to bind free PEl and PEI-siRNA complex. Then, the solution was transferred into Ultra Centrifugal Filters (molecular weight cut-off 50 $\mathrm{kDa}$ ), and then centrifuged under $5000 \mathrm{rpm}$ at $4^{\circ} \mathrm{C}$ for $30 \mathrm{~min}$. FAM-labeled siRNA (Ex $488 \mathrm{~nm}, \mathrm{Em} 520$ $\mathrm{nm}$ ) in the filtrate was collected for the quantification of nucleic acid release.

\section{Antitumor Therapy in Orthotopic Colorectal Tumor Model}

The orthotopic CRC model was developed in female BALB/c nude mice as described previously ${ }^{44} .28$ day after orthotopic tumor cell (HCT116-luc, COLO205-luc) inoculation, the tumor-bearing mice were randomly allocated into five groups $(n=5)$ according to the tumor bioluminescent intensity and treated with saline (control), MSN loaded with negative control siRNA with a scrambled sequence (siNC@MSN), and MSN loaded with siPum1 (siPum1@MSN), siPum2 (siPum1@MSN) or both (siPum1/2@MSN). The siRNA dose was $1 \mathrm{mg} / \mathrm{kg}$ on days 0, 3, 6, and 9, respectively. Each group included 10 mice. The tumor burden was monitored weekly using bioluminescence imaging (IVIS spectrum CT). The body weight was recorded throughout the study. At the end of the study (day 30), the mice were sacrificed, the tumors were removed and weighted. Other major organs included heart, liver, spleen, lung, and kidney were harvested for ex vivo bioluminescent imaging to examine the metastasis. In a separate study, on day 11 ( $2 \mathrm{~d}$ after the last injection of the nanoparticles), three mice from each group were sacrificed. The major organs, including heart, liver, spleen, lung, and kidney were collected for H\&E histological assay for toxicity evaluation.

\section{Accession Numbers}

The accession number for the RNA-seq and PAR-CLIP data reported in this paper is GEO: PRJNA648706.

\section{Declarations}

\section{Conflict of interest}

The authors have no conflicting financial interests

\section{Funding}

This research was funded by the Shanghai Institute for Advanced Immunochemical Studies at ShanghaiTech University. S.L. was supported by National Natural Science Foundation of China (81772798). C.F. was supported by National Natural Science Foundation of China (81773274). Y.G., and Z.L. were supported by the School of Life Science and Technology at ShanghaiTech University.

\section{Author contributions}


H.L. and S.L. planned the project. Y.G., Z.L., Y.Y., C.F., S.L., and H.L. designed the experiments and interpreted the results. In details, Y.G. performed the AOM-DSS mouse experiments and S.L. did data analysis. Y.G. and Z.L. performed the experiments on Pum1/2 biological function in vitro and data analysis. Y.G. performed the experiments of PAR-CLIP, Z.Y. and Y.G. performed analyses of PAR-CLIP data. Z.L. performed RNA seq and mass spec experiments, Z.Y., Y.G., S.L., and W.W. did data analysis. Y.G. and J.Z. performed Pum1 target gene validation experiments and data analysis. Y.G. generated p21 Pumilio binding site mutations and studied their biological function. Y.Y. performed RNAi therapy experiments and data analysis. S.L. analyzed public cancer database. Y.G., S.L., C.F., and H.L. wrote the manuscript. Z.L., and Z.Y. commented on the manuscript. H.L. and S.L. supervised the overall work and C.F. was responsible for RNAi therapy.

\section{Acknowledgments}

We thank Wenzhang Chen, Hongwei Zhang in the ShanghaiTech University Proteomics platform for assistance with mass spectrometry; Peixiang Ma, Fan Yang, Shuo Shi, Chen Wang, Ting Lu in the SIAIS at ShanghaiTech University for technical asistance. We appreciate ShanghaiTech High Performance Computing Platform for providing the computing resources and technical support.

\section{References}

1. Bray F, Ferlay J, Soerjomataram I, et al. Global cancer statistics 2018: GLOBOCAN estimates of incidence and mortality worldwide for 36 cancers in 185 countries. CA Cancer J Clin 2018;68:394424.

2. Fearon ER. Molecular genetics of colorectal cancer. Annu Rev Pathol 2011;6:479-507.

3. Cancer Genome Atlas N. Comprehensive molecular characterization of human colon and rectal cancer. Nature 2012;487:330-7.

4. Chatterji P, Rustgi AK. RNA Binding Proteins in Intestinal Epithelial Biology and Colorectal Cancer. Trends Mol Med 2018;24:490-506.

5. Pereira B, Billaud M, Almeida R. RNA-Binding Proteins in Cancer: Old Players and New Actors. Trends Cancer 2017;3:506-528.

6. Spassov DS, Jurecic R. The PUF family of RNA-binding proteins: does evolutionarily conserved structure equal conserved function? IUBMB Life 2003;55:359-66.

7. Van Etten J, Schagat TL, Hrit J, et al. Human Pumilio proteins recruit multiple deadenylases to efficiently repress messenger RNAs. J Biol Chem 2012;287:36370-83.

8. Zamore PD, Williamson JR, Lehmann R. The Pumilio protein binds RNA through a conserved domain that defines a new class of RNA-binding proteins. Rna 1997;3:1421-33.

9. Miller MA, Olivas WM. Roles of Puf proteins in mRNA degradation and translation. Wiley Interdiscip Rev RNA 2011;2:471-92. 
10. Lin H, Spradling AC. A novel group of pumilio mutations affects the asymmetric division of germline stem cells in the Drosophila ovary. Development 1997;124:2463-76.

11. Doniach T, Hodgkin J. A sex-determining gene, fem-1, required for both male and hermaphrodite development in Caenorhabditis elegans. Dev Biol 1984;106:223-35.

12. Barton MK, SchedI TB, Kimble J. Gain-of-function mutations of fem-3, a sex-determination gene in Caenorhabditis elegans. Genetics 1987;115:107-19.

13. Chen $D$, Zheng W, Lin A, et al. Pumilio 1 suppresses multiple activators of $p 53$ to safeguard spermatogenesis. Curr Biol 2012;22:420-5.

14. Mak W, Fang C, Holden T, et al. An Important Role of Pumilio 1 in Regulating the Development of the Mammalian Female Germline. Biol Reprod 2016;94:134.

15. Mak W, Xia J, Cheng EC, et al. A role of Pumilio 1 in mammalian oocyte maturation and maternal phase of embryogenesis. Cell Biosci 2018;8:54.

16. Zhang $M$, Chen $D$, Xia J, et al. Post-transcriptional regulation of mouse neurogenesis by Pumilio proteins. Genes Dev 2017;31:1354-1369.

17. Galgano A, Forrer M, Jaskiewicz L, et al. Comparative analysis of mRNA targets for human PUFfamily proteins suggests extensive interaction with the miRNA regulatory system. PLoS One 2008;3:0003164.

18. Hafner $M$, Landthaler $M$, Burger $L$, et al. Transcriptome-wide identification of RNA-binding protein and microRNA target sites by PAR-CLIP. Cell 2010;141:129-41.

19. Morris AR, Mukherjee N, Keene JD. Ribonomic analysis of human Pum1 reveals cis-trans conservation across species despite evolution of diverse mRNA target sets. Mol Cell Biol 2008;28:4093-103.

20. Naudin C, Hattabi A, Michelet F, et al. PUMILIO/FOXP1 signaling drives expansion of hematopoietic stem/progenitor and leukemia cells. 2017;129:2493-2506.

21. Xia L, Yan Q, Sun Q, et al. MiR-411-5p acts as a tumor suppressor in non-small cell lung cancer through targeting PUM1. 2018;22:5546-5553.

22. Dai $\mathrm{H}$, Shen $\mathrm{K}$, Yang $\mathrm{Y}$, et al. PUM1 knockdown prevents tumor progression by activating the PERK/elF2/ATF4 signaling pathway in pancreatic adenocarcinoma cells. Cell Death Dis 2019;10:595.

23. Guan X, Chen S, Liu Y, et al. PUM1 promotes ovarian cancer proliferation, migration and invasion. 2018;497:313-318.

24. Lee S, Kopp F, Chang TC, et al. Noncoding RNA NORAD Regulates Genomic Stability by Sequestering PUMILIO Proteins. Cell 2016;164:69-80.

25. Miles WO, Tschop K, Herr A, et al. Pumilio facilitates miRNA regulation of the E2F3 oncogene. Genes Dev 2012;26:356-68.

26. Hong Y, Downey T, Eu KW, et al. A 'metastasis-prone' signature for early-stage mismatch-repair proficient sporadic colorectal cancer patients and its implications for possible therapeutics. Clinical 
\& Experimental Metastasis 2010;27:83-90.

27. Skrzypczak M, Goryca K, Rubel T, et al. Modeling oncogenic signaling in colon tumors by multidirectional analyses of microarray data directed for maximization of analytical reliability. PLoS One 2010;5.

28. Uyhazi KE, Yang Y, Liu N, et al. Pumilio proteins utilize distinct regulatory mechanisms to achieve complementary functions required for pluripotency and embryogenesis. Proc Natl Acad Sci U S A 2020;117:7851-7862.

29. Tanaka T, Kohno H, Suzuki R, et al. A novel inflammation-related mouse colon carcinogenesis model induced by azoxymethane and dextran sodium sulfate. Cancer Sci 2003;94:965-73.

30. Ran FA, Hsu PD, Lin CY, et al. Double nicking by RNA-guided CRISPR Cas9 for enhanced genome editing specificity. Cell 2013;154:1380-9.

31. Wiedemeyer R, Brennan C, Heffernan TP, et al. Feedback circuit among INK4 tumor suppressors constrains human glioblastoma development. Cancer Cell 2008;13:355-364.

32. Eastham JA, Hall SJ, Sehgal I, et al. In vivo gene therapy with p53 or p21 adenovirus for prostate cancer. Cancer Res 1995;55:5151-5.

33. Bai F, Chan HL, Smith MD, et al. p19(INk4d) Is a Tumor Suppressor and Controls Pituitary Anterior Lobe Cell Proliferation. Molecular and Cellular Biology 2014;34:2121-2134.

34. Krimpenfort P, IJpenberg A, Song JY, et al. p15(Ink4b) is a critical tumour suppressor in the absence of p16(Ink4a). Nature 2007;448:943-U11.

35. Corcoran DL, Georgiev S, Mukherjee N, et al. PARalyzer: definition of RNA binding sites from PARCLIP short-read sequence data. Genome Biology 2011;12.

36. Kedde M, van Kouwenhove M, Zwart W, et al. A Pumilio-induced RNA structure switch in p27-3' UTR controls miR-221 and miR-222 accessibility. Nat Cell Biol 2010;12:1014-20.

37. Jiang P, Singh M, Coller HA. Computational assessment of the cooperativity between RNA binding proteins and MicroRNAs in Transcript Decay. PLoS Comput Biol 2013;9:e1003075.

38. HafezQorani S, Lafzi A, de Bruin RG, et al. Modeling the combined effect of RNA-binding proteins and microRNAs in post-transcriptional regulation. Nucleic Acids Res 2016;44:2.

39. Friend K, Campbell ZT, Cooke A, et al. A conserved PUF-Ago-eEF1A complex attenuates translation elongation. Nat Struct Mol Biol 2012;19:176-83.

40. Rudel S, Wang Y, Lenobel R, et al. Phosphorylation of human Argonaute proteins affects small RNA binding. Nucleic Acids Res 2011;39:2330-43.

41. Liu J, Carmell MA, Rivas FV, et al. Argonaute2 is the catalytic engine of mammalian RNAi. Science 2004;305:1437-41.

42. Lewis BP, Burge CB, Bartel DP. Conserved seed pairing, often flanked by adenosines, indicates that thousands of human genes are microRNA targets. Cell 2005;120:15-20.

43. Kara M, Yumrutas O, Ozcan O, et al. Differential expressions of cancer-associated genes and their regulatory miRNAs in colorectal carcinoma. Gene 2015;567:81-6. 
44. Liu H-J, Luan X, Feng H-Y, et al. Integrated Combination Treatment Using a "Smart" Chemotherapy and MicroRNA Delivery System Improves Outcomes in an Orthotopic Colorectal Cancer Model. Advanced Functional Materials 2018;28:1801118.

45. Lu Y, Aimetti AA, Langer R, et al. Bioresponsive materials. Nature Reviews Materials 2016;2:16075.

46. Talelli M, Barz M, Rijcken CJF, et al. Core-crosslinked polymeric micelles: Principles, preparation, biomedical applications and clinical translation. Nano Today 2015;10:93-117.

47. Leung U, Gönen M, Allen PJ, et al. Colorectal cancer liver metastases and concurrent extrahepatic disease treated with resection. Annals of surgery 2017;265:158.

48. Tomlinson JS, Jarnagin WR, DeMatteo RP, et al. Actual 10-year survival after resection of colorectal liver metastases defines cure. Journal of Clinical Oncology 2007;25:4575-4580.

49. Fernandez S, Risolino M, Mandia N, et al. miR-340 inhibits tumor cell proliferation and induces apoptosis by targeting multiple negative regulators of p27 in non-small cell lung cancer. Oncogene 2015;34:3240-50.

50. Naudin C, Hattabi A, Michelet F, et al. PUMILIO/FOXP1 signaling drives expansion of hematopoietic stem/progenitor and leukemia cells. Blood 2017;129:2493-2506.

51. Brocard M, Khasnis S, Wood CD, et al. Pumilio directs deadenylation-associated translational repression of the cyclin-dependent kinase 1 activator RGC-32. Nucleic Acids Res 2018;46:37073725.

52. Guan X, Chen S, Liu Y, et al. PUM1 promotes ovarian cancer proliferation, migration and invasion. Biochem Biophys Res Commun 2018;497:313-318.

53. Miles WO, Lembo A, Volorio A, et al. Alternative Polyadenylation in Triple-Negative Breast Tumors Allows NRAS and c-JUN to Bypass PUMILIO Posttranscriptional Regulation. Cancer Res 2016;76:7231-7241.

54. Narita R, Takahasi K, Murakami E, et al. A novel function of human Pumilio proteins in cytoplasmic sensing of viral infection. PLoS Pathog 2014;10:e1004417.

55. Ponten F, Jirstrom K, Uhlen M. The Human Protein Atlas-a tool for pathology. J Pathol 2008;216:387-93.

56. Liu S, Wang Z, Liu Z, et al. miR-221/222 activate the Wnt/beta-catenin signaling to promote triplenegative breast cancer. J Mol Cell Biol 2018;10:302-315.

57. Wang Z, Liu N, Shi S, et al. The Role of PIWIL4, an Argonaute Family Protein, in Breast Cancer. J Biol Chem 2016;291:10646-58.

58. Langmead B, Trapnell C, Pop M, et al. Ultrafast and memory-efficient alignment of short DNA sequences to the human genome. Genome Biol 2009;10:R25.

59. Li H, Handsaker B, Wysoker A, et al. The Sequence Alignment/Map format and SAMtools. Bioinformatics 2009;25:2078-9.

60. Corcoran DL, Georgiev S, Mukherjee N, et al. PARalyzer: definition of RNA binding sites from PARCLIP short-read sequence data. Genome Biol 2011;12:R79. 
61. Quinlan AR, Hall IM. BEDTools: a flexible suite of utilities for comparing genomic features. Bioinformatics 2010;26:841-2.

62. Robinson JT, Thorvaldsdottir H, Winckler W, et al. Integrative genomics viewer. Nat Biotechnol 2011;29:24-6.

\section{Figures}
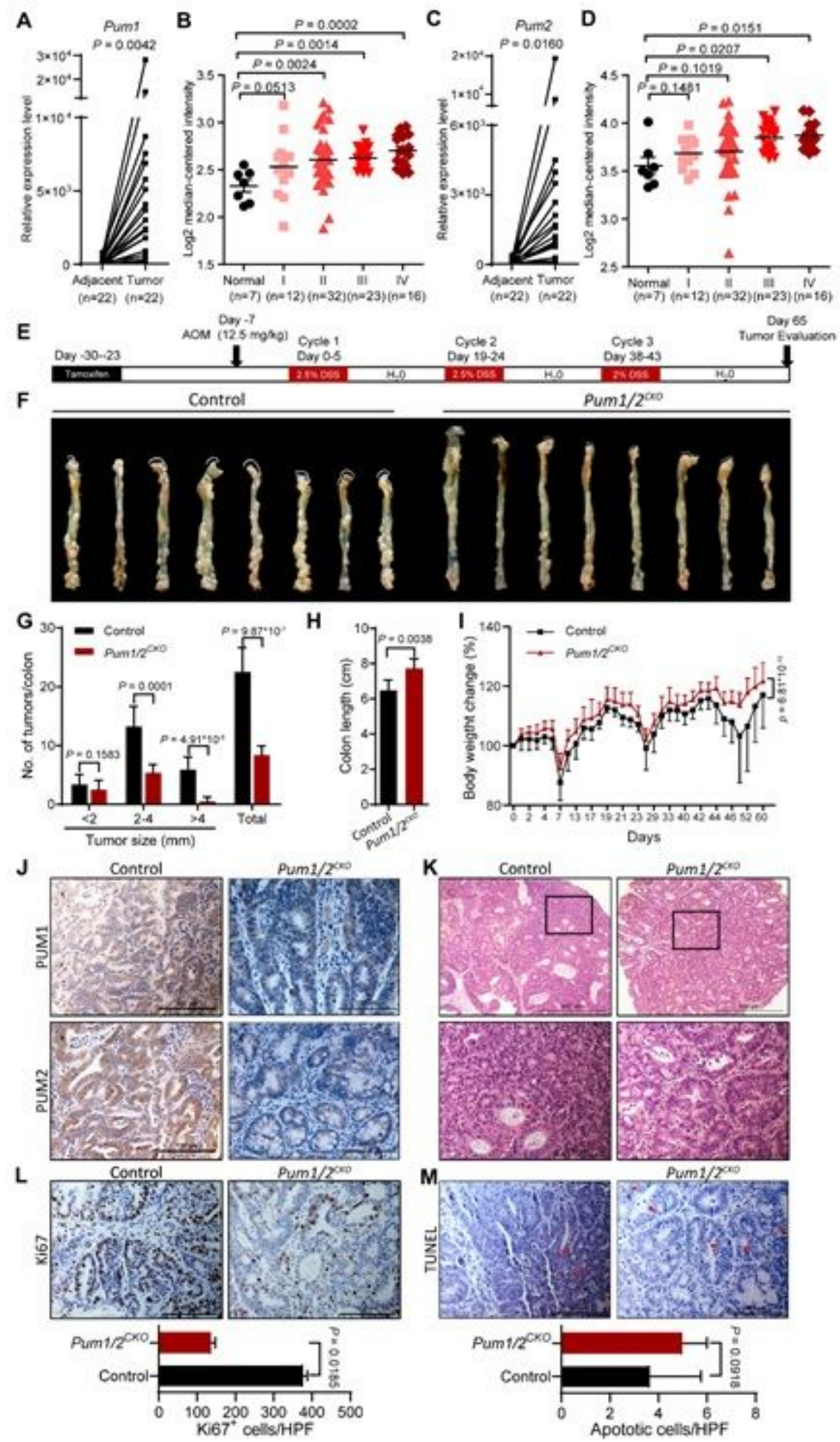


\section{Figure 1}

Pum1/2CKO suppresses the development of colorectal cancer. (A and C) Expression of Pum1 (A) or Pum2 (C) in human colorectal cancer clinical specimens from 22 patients as compared to adjacent tissues. (B and D) Expression of Pum1 (B) or Pum2 (G) in human colorectal cancer clinical specimens at different stages using the TCGA mRNA HiSeq expression array data. (E) Schematic overview of the colorectal cancer induction model. C57BL/6J mice ( $n=8$ for each group) were injected with tamoxifen for 7 days (injection every other day) to knock out Pum $1 / 2$ in intestinal epithelial cells, then 16 days later, the mice were injected with azoxymethane (AOM), followed by 3 cycles of treatment with DSS, as described in experimental procedures. $(F)$ The colon and rectum of control (Pum1flox/flox::Pum2flox/flox) and Pum1/2CKO (Lgr5cre::Pum1flox/flox::Pum2flox/flox) mice in AOM / DSS model. (G) The number of tumors in the entire colon and rectum, and diameter of the tumors were measured at the end of the study. $(\mathrm{H})$ The colon length of control and Pum1/2CKO mice in AOM / DSS model. (I) Weight changes of control and Pum1/2CKO mice in AOM / DSS model. (J) Immunohistochemistry staining for PUM1 and PUM2 in colon tissues containing tumors from control and Pum1/2CKO mice in AOM / DSS model. (K) H\&E staining of colon tumor in control and Pum1/2CKO mice in AOM / DSS model. ( $L$ and $M$ ) Representative immunostaining for Ki67 ( $L$ ) and TUNEL staining $(M)$ in tumors from control and Pum1/2CKO mice in AOM / DSS model. 

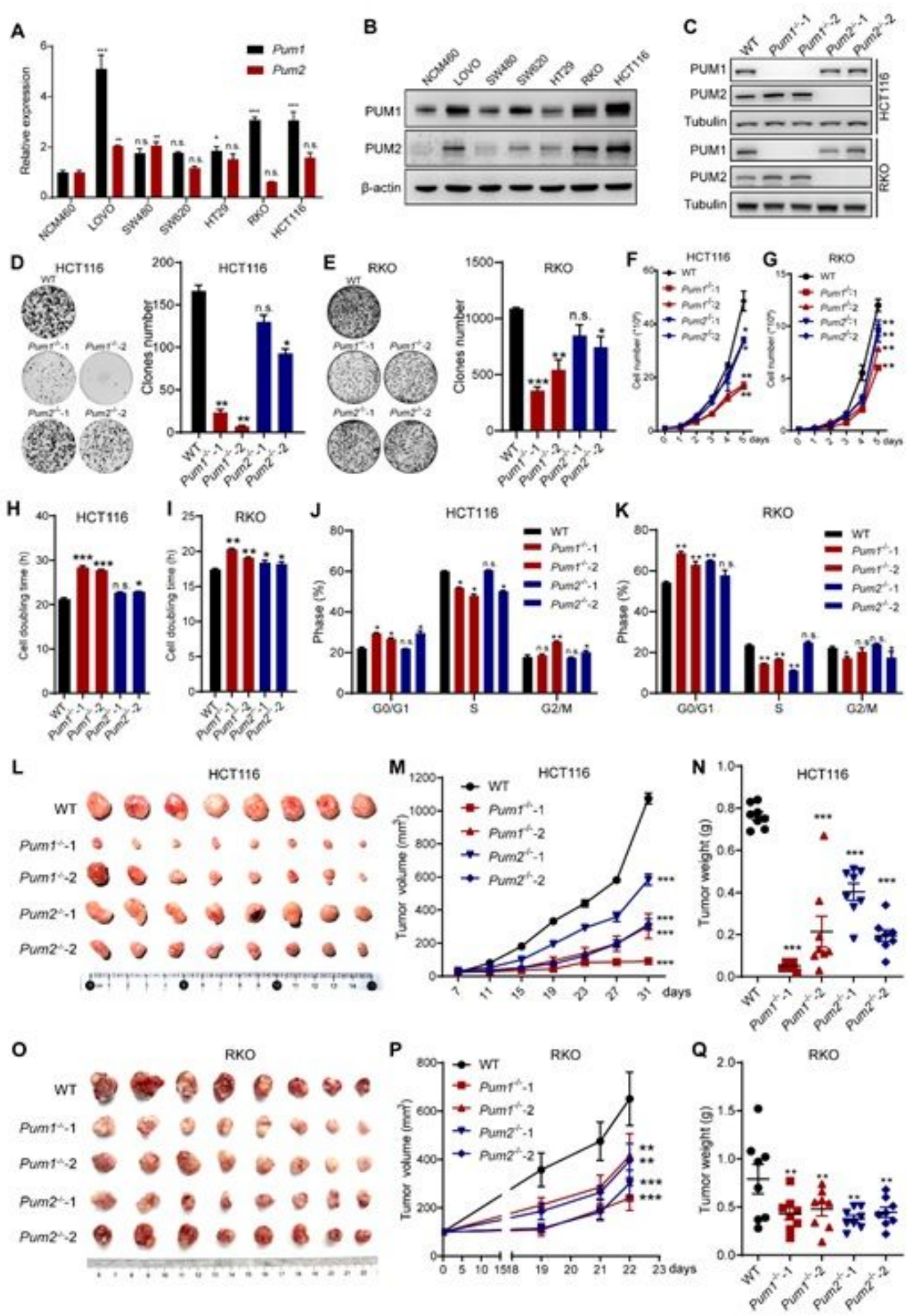

Figure 2

PUM1 and PUM2 drive colorectal cancer cell growth in vitro and tumorigenicity in vivo. (A and B) mRNA (A) and protein (B) levels of PUM1 or PUM2 in different colorectal cancer cell lines. (C) Knockout efficiency of Pum1 and Pum2 in HCT116 and RKO cells. (D and E) Colony formation assay of WT, Pum1-/- or Pum2-/- HCT116 (D) and RKO (E) cells. Colony numbers were counted after 10 days. (F and G) Growth curve of WT, Pum1-/- or Pum2-/- HCT116 (F) and RKO (G) cells. (H and I) Cell doubling time of 
WT, Pum1-/- or Pum2-/- HCT116 (H) and RKO (I) cells. (J and K) Cell cycle analysis of WT, Pum1-/- or Pum2-/- HCT116 (J) and RKO (K) cells. (L-N) Tumor growth (L), tumor volume $(\mathrm{M})$, tumor weight $(\mathrm{N})$ in mice injected with WT, Pum1-/- or Pum2-/- HCT116 cells. (O-Q) Tumor growth (0), tumor volume (P), tumor weight (Q) in mice injected with WT, Pum1-/- or Pum2-/- RKO cells. ${ }^{\star} \mathrm{P}<0.05,{ }^{\star *} \mathrm{P}<0.01$, ${ }^{\star \star \star} \mathrm{P}<$ 0.001 .
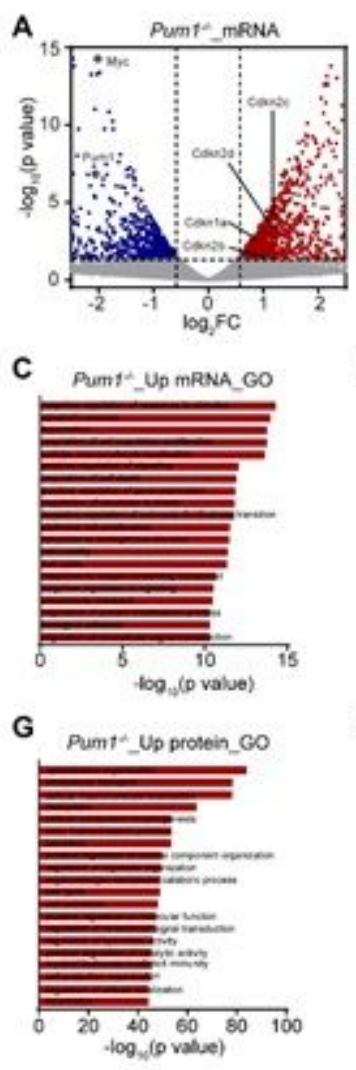
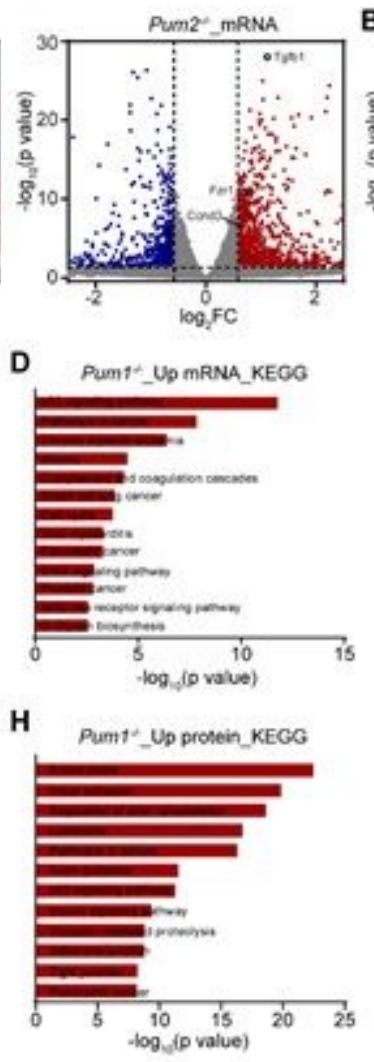
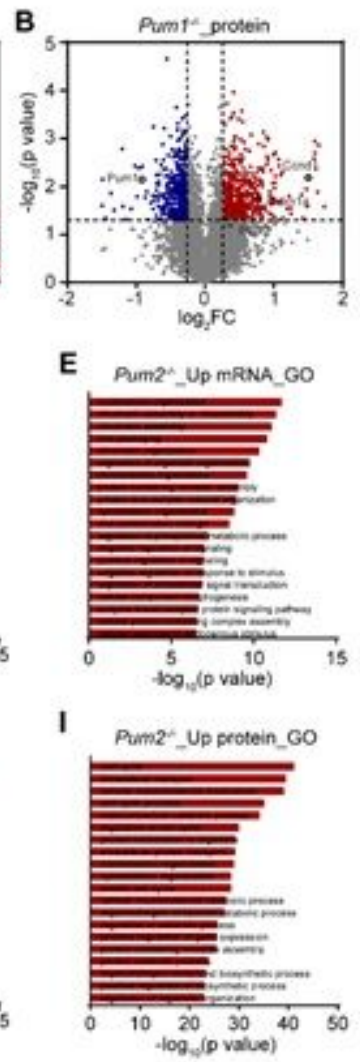
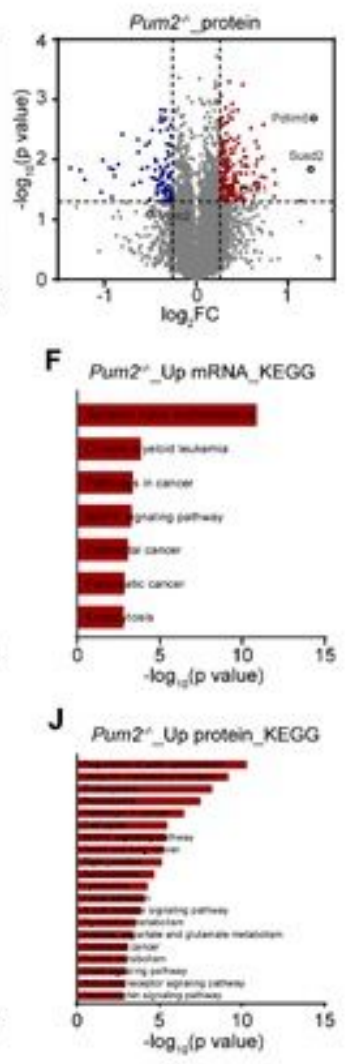

\section{Figure 3}

Cell cycle related genes are mainly affected in Pum1-/- HCT116 cells. (A and B) Volcano plot showing the mRNA (A) or protein (B) changes between WT and Pum1-/- or Pum2-/- samples. Regulated genes including those in cell cycle, Pum1, and Pum2 are labeled. (C and D) GO (C) and KEGG (D) analysis of upregulated mRNAs in Pum1-/- HCT116 cells. (E and F) GO (E) and KEGG (F) analysis of upregulated mRNAs in Pum2-/- HCT116 cells. ( $G$ and $H)$ GO $(G)$ or KEGG $(H)$ analysis of upregulated proteins in Pum1-/- HCT116 cells. (I and J) GO (I) or KEGG (J) analysis of upregulated proteins in Pum2-/- HCT116 cells. 
A
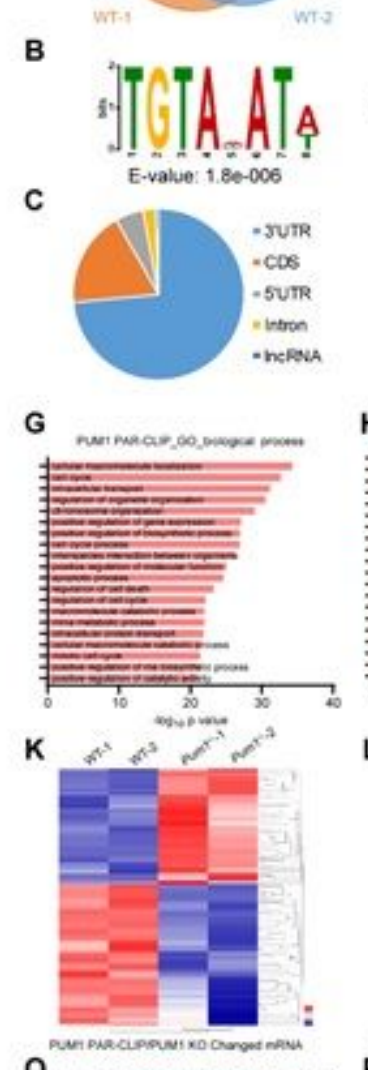

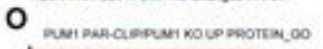

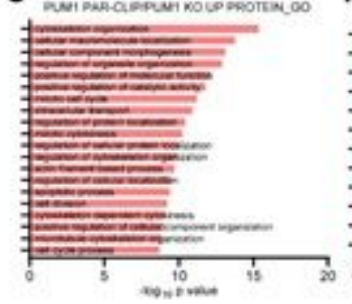

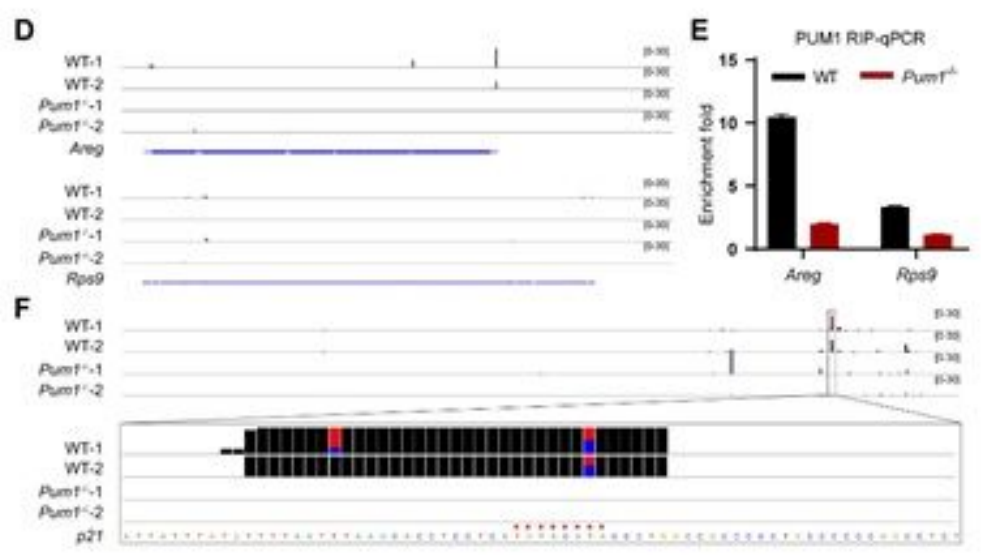
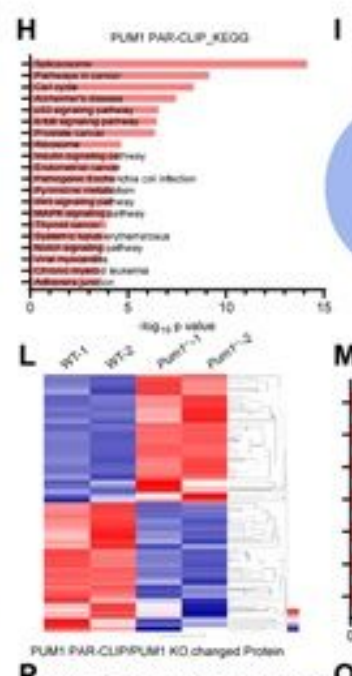

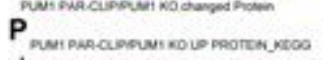

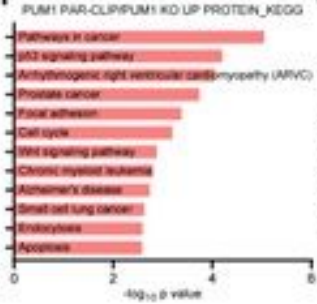

1 J

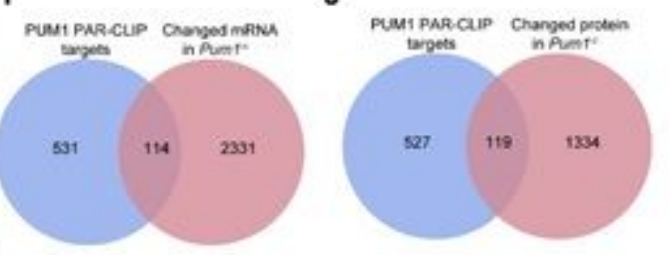

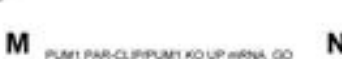
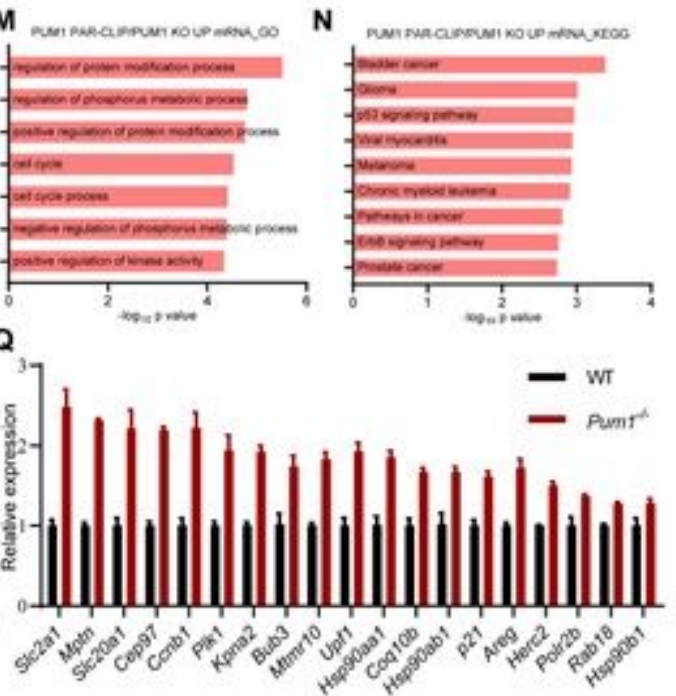

Figure 4

PAR-CLIP-seq (PAR-CLIP combined with high-throughput sequencing) identification of PUM1 targets. (A) Reproducibility of two biological replicates for PUM1 PAR-CLIP. (B) MEME results for de novo discovery of the binding motif for PUM1. (C) Distribution of the PUM1-binding sites in different genomic sections. (D) PUM1 PAR-CLIP peaks on the Areg and Rps9 transcript in WT and Pum1-/- HCT116 cells. Git1 is a target for PUM1, and Rps9 is a nontarget. (E) PUM1 RIP-qPCR validation of the target Areg and nontarget Rps9. (F) PUM1 PAR-CLIP peaks on the p21 transcript in WT and Pum1-/- HCT116 cells. TGTACATA is binding site of PUM1. (G and H) GO and KEGG analysis of PUM1 PAR-CLIP targets in HCT116 cells. (I and J) A venn diagram showing the overlap of PUM1 PAR-CLIP targets with changed mRNAs $(I)$ or proteins $(\mathrm{J})$ in Pum1-/- HCT116 cells. ( $K$ and $L$ ) Heatmap showing the mRNAs $(K)$ and proteins $(L)$ expression of PUM1 PAR-CLIP targets in Pum1-/- HCT116 cells. (M-P) GO or KEGG analysis of PUM1 PAR-CLIP target mRNAs 
$(\mathrm{M}, \mathrm{N})$ or proteins $(\mathrm{O}, \mathrm{P})$ that are upregulated in Pum1-/- HCT116 cells, demonstrating enrichment of genes involved in cell cycle. (Q) qRT-PCR validation of overlap genes of PUM1 PAR-CLIP targets with upregulated mRNAs in Pum1-/- HCT116 cells. All genes shown were significantly upregulated in Pum1-/HCT116 cells $(P<0.05)$.
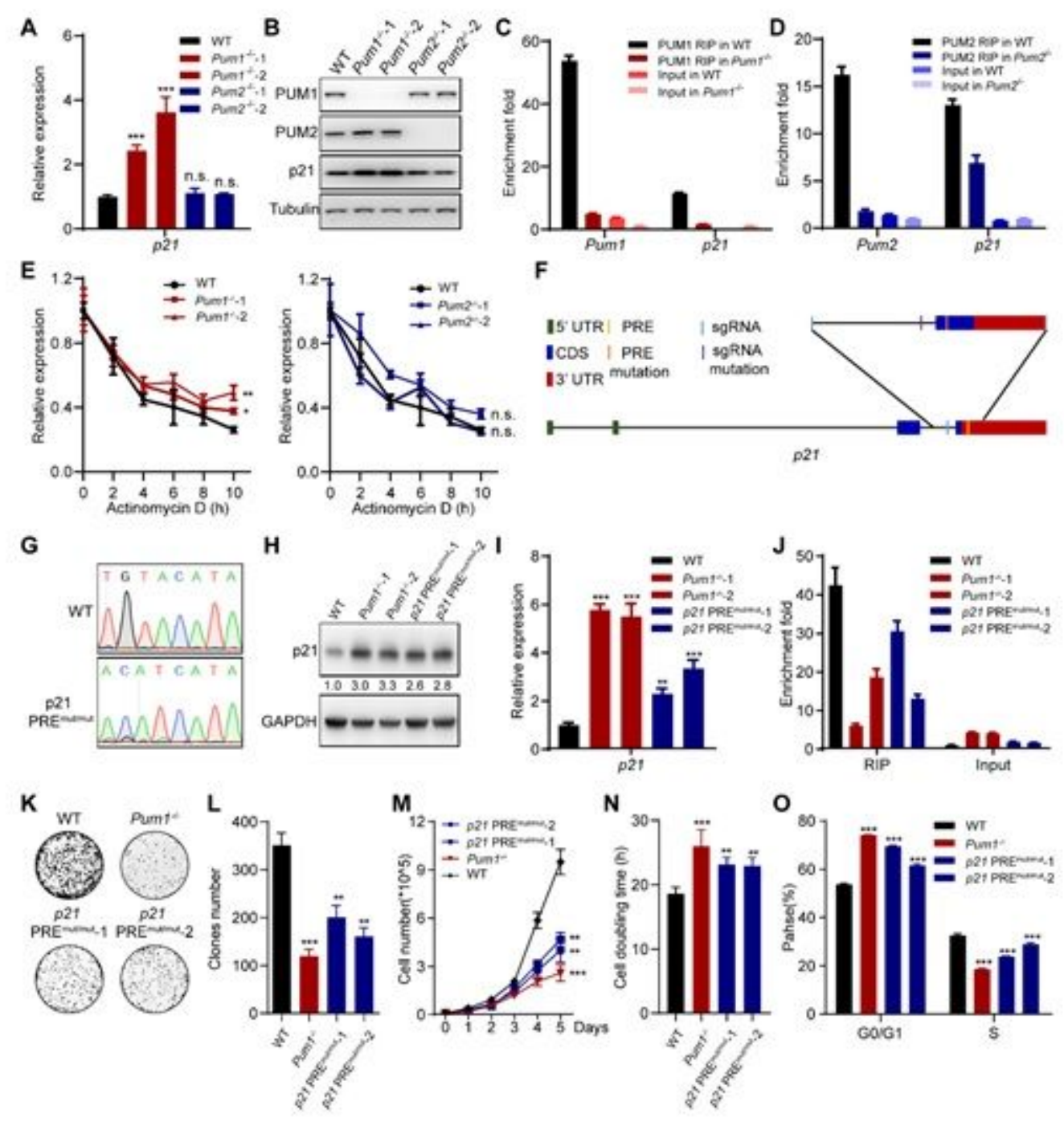

\section{Figure 5}

PUM1 regulates colorectal cancer cell growth through directly repressing p21. (A and B) The mRNA (A) and protein (B) levels of p21 in Pum1-/- or Pum2-/- HCT116 cells. (C and D) Enrichment of p21 with PUM1 RIP or PUM2 RIP in WT, Pum1-/- or Pum2-/- HCT116 cells. (E) The p21 mRNA levels in WT, Pum1-/or Pum2-/- HCT116 cells treated with actinomycin $D(10 \mathrm{mg} / \mathrm{mL})$ for the indicated period. (F) Schematic diagram of p21 PRE mutations generation in HCT116 cells. (G) The representative sequencing results of PRE in WT and p21 PREmut/mut cells. ( $\mathrm{H}$ and $\mathrm{I}$ ) The P21 mRNA (I) and protein $(\mathrm{H})$ levels in WT, Pum1-/and p21 PREmut/mut HCT116 cells. (J) Enrichment of p21 with PUM1 RIP in WT, Pum1-/- and p21 PREmut/mut HCT116 cells. (K) Colony formation assay of WT and p21 PREmut/mut HCT116 cells. (L) Colony numbers of WT and p21 PREmut/mut HCT116 cells. (M) Growth curve of WT and p21 
PREmut/mut HCT116 cells. (N) Cell doubling time of WT and p21 PREmut/mut HCT116 cells. (0) Cell cycle analysis of WT and p21 PREmut/mut HCT116 cells.

A

pursic.
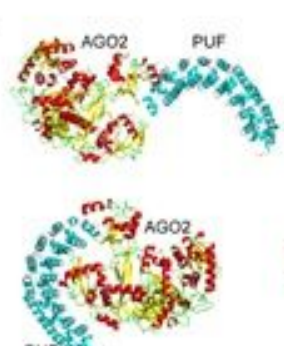
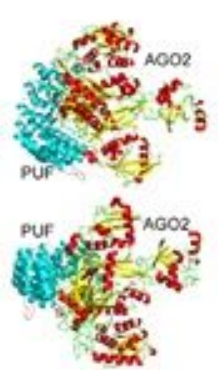
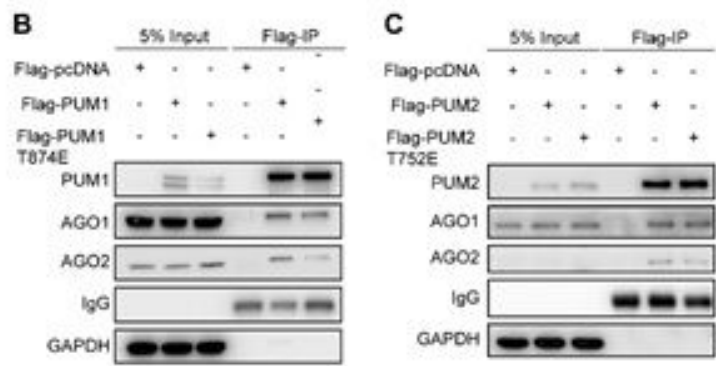

D

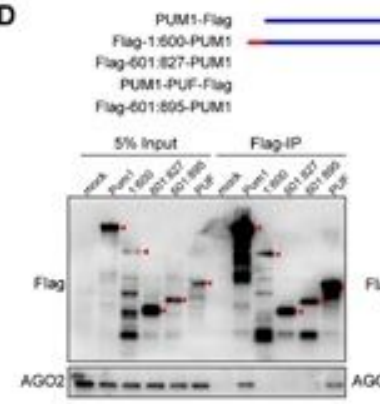

G

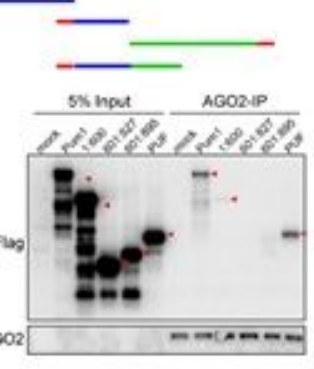

E PUM PUM2

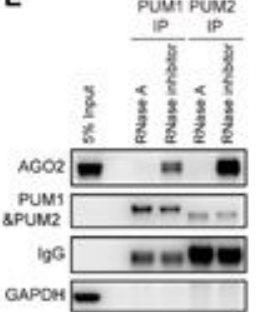

$\mathbf{F}$
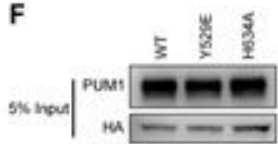

pumis $\mid \begin{aligned} & \text { PuMn } \\ & \mathrm{man}\end{aligned}$

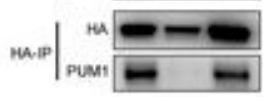

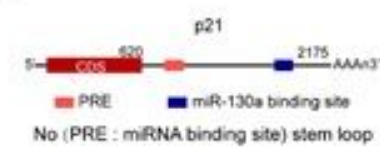

J

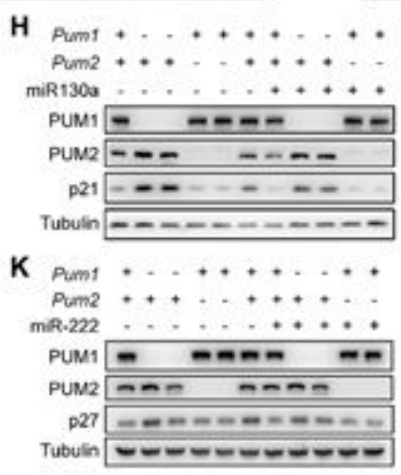

M

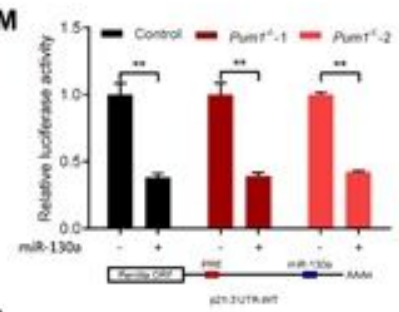

N
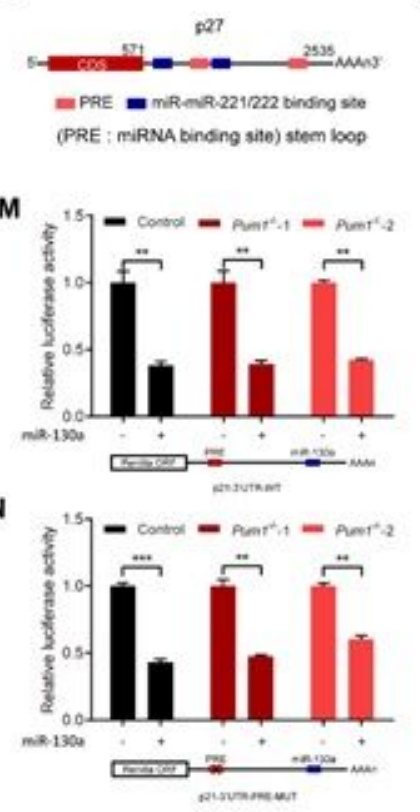

0

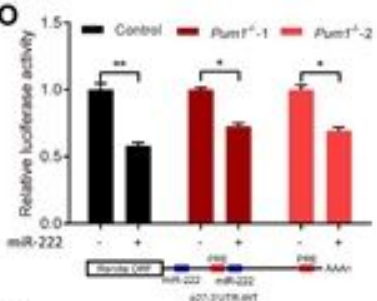

Q

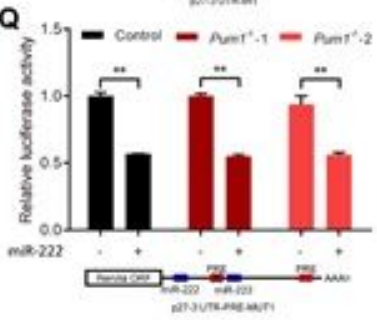

I

Pumt +...+.+...+.

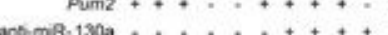

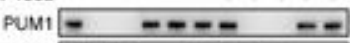

PUM2 $-\infty-\infty$

p21

Tubulin

L

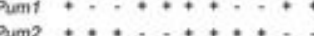

antim $\mathrm{R} \cdot 222,+\ldots+\ldots+\ldots+\ldots+$

PUM1 $A \quad B A B A+B R$

PUM2

p27

Tubulin $-0-1-0-1-0$

P

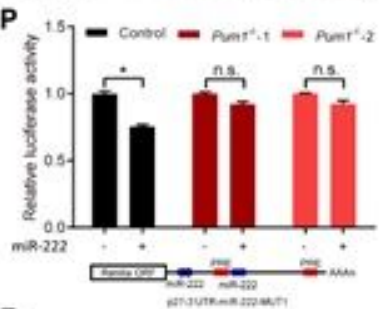

R

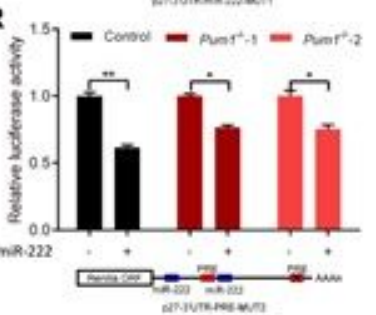

Figure 6

PUM1 represses p21 and p27 expression in different regulatory ways. (A) Predicted interaction mode of PUF and AG02. (B) The T874E mutation of PUM1 partially blocked the binding of PUM1 protein to AG02 protein. (C) The T752E mutation of PUM2 partially blocked the binding of PUM2 protein to AGO2 protein. 
(D) Co-IP analyses of PUM1-Flag and PUM1 truncated expression vectors in HCT116 cells and cell lysates were immunoprecipitated with Flag antibody (left). Co-IP analyses of AGO2 and PUM1 truncated expression vectors in HCT116 cells and cell lysates were immunoprecipitated with AGO2 antibody (right). (E) Co-IP analyses of PUM1, PUM2 and AGO2 with RNase A or RNase inhibitor treatment in HCT116 cells and cell lysates were immunoprecipitated with PUM1 or PUM2 antibody. (F) Co-IP analyses of HA-AGO2Y529E, HA-AG02-H634A and PUM1 in HCT116 cells and cell lysates were immunoprecipitated with PUM1 or HA antibodies. Y529E: AGO2 miRNA binding mutant. H634A: AGO2 slicer activity mutant. (G) Schematic diagram of PUMILIO and miR-130a regulating p21. (H and I) PUM1, PUM2 and p21 protein levels in the indicated samples. (J) Schematic diagram of PUMILIO and miR-221/222 regulating p27 in both (stem-loop) manner. (K and L) PUM1, PUM2 and p27 protein levels in the indicated samples. ( $M$ and N) The luciferase activity of the p21 3'UTR reporter in WT, Pum1-/- HCT116 cells transfected with $100 \mathrm{nM}$ miR-NC or miR-130a mimics, $100 \mathrm{ng}$ of the psiCHECK-p21 3'UTR reporter plasmid (N) or psiCHECK-p21 3'UTR-PRE-MUT reporter plasmid (O) for 48 hours. (O-R) The luciferase activity of the p27 3'UTR reporter in WT, Pum1-/- HCT116 cells transfected with $100 \mathrm{nM}$ miR-NC or miR-222 mimics, $100 \mathrm{ng}$ of the psiCHECK-p27 3'UTR-WT reporter plasmid (0), psiCHECK-p27 3'UTR-miR-222-MUT1 reporter plasmid (P), psiCHECK-p27 3'UTR-PRE-MUT1 reporter plasmid (Q) or psiCHECK-p27 3'UTR-PRE-MUT2 reporter plasmid (R) for 48 hours. ${ }^{*} P<0.05, * * P<0.01, * * * P<0.001$. 
A
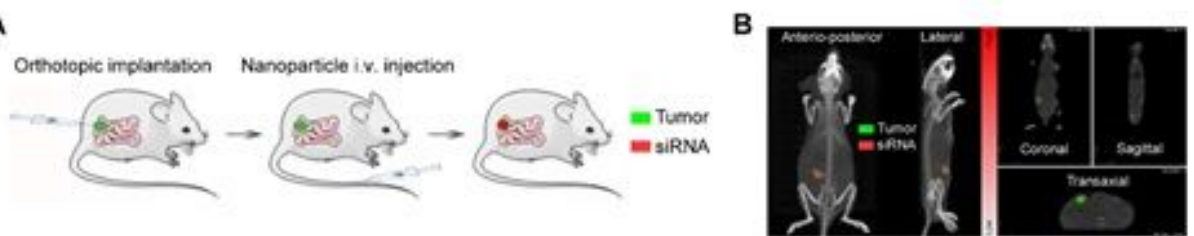

c

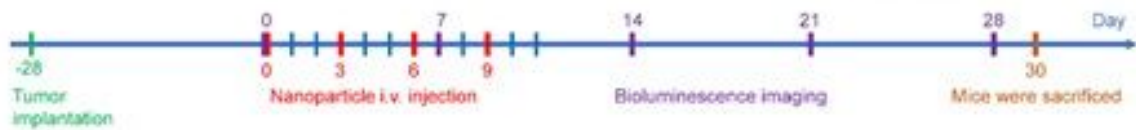

D

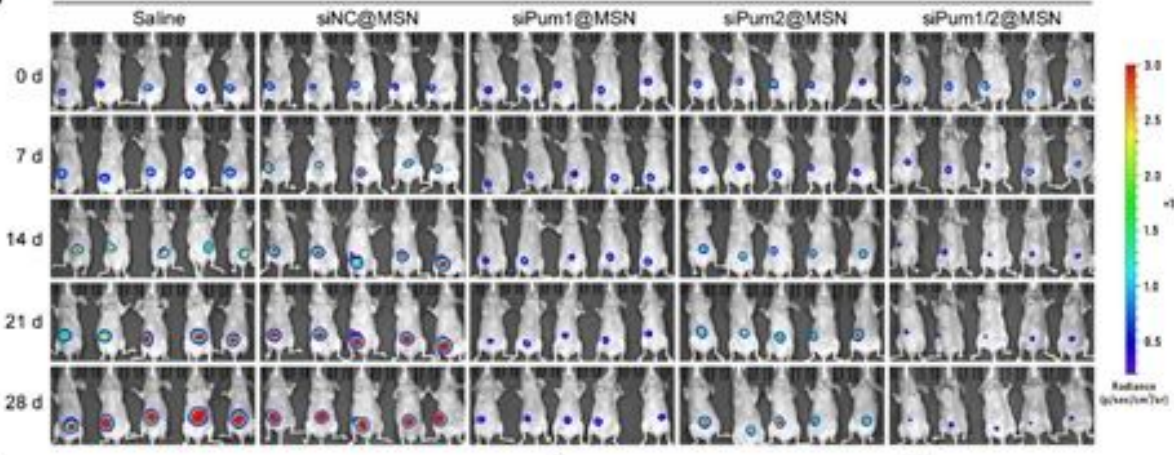

E
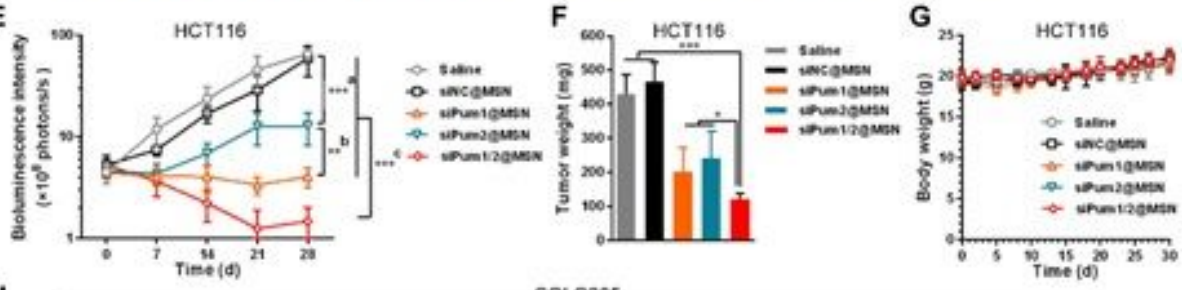

H
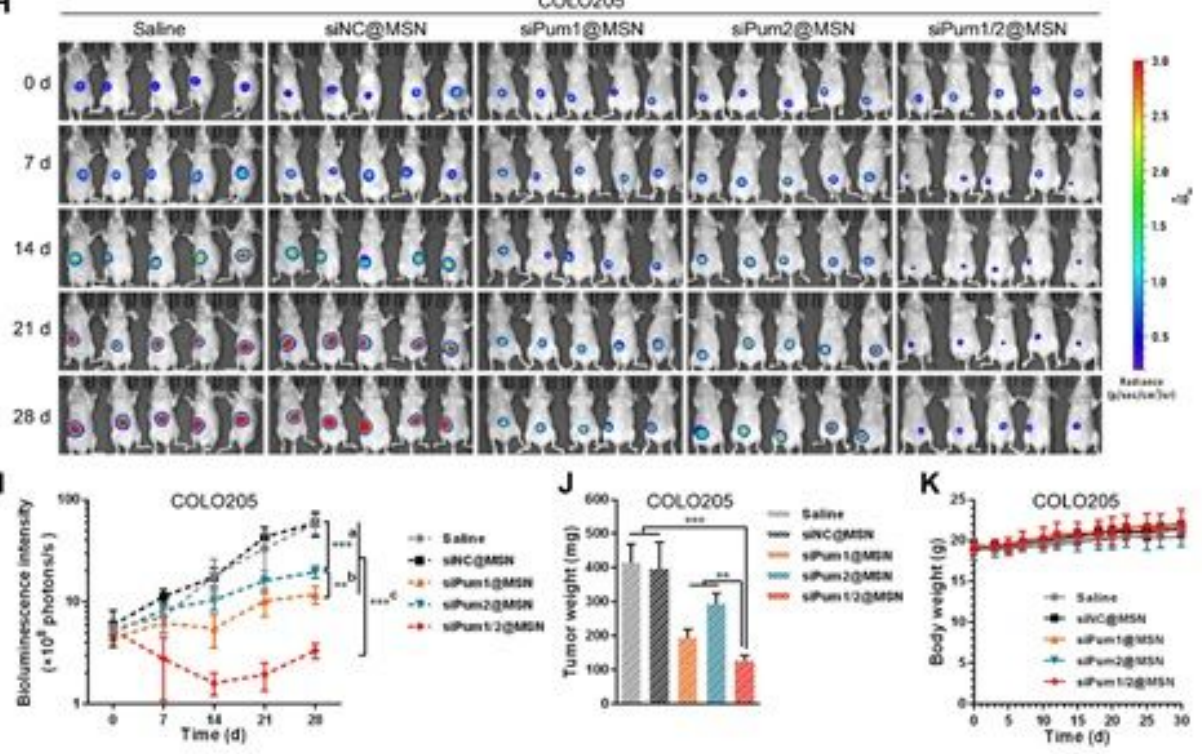

Figure 7

In vivo therapy using siRNA-loaded MSN in mice bearing orthotopic HCT116 or COLO205 colorectal tumor. (A) Schematic diagram of orthotopic implantation and nanoparticle therapy. (B) Tumor targeting of the siRNA-loaded nanoparticles was observed using IVIS Spectrum CT multimodal imaging system. Luciferase labeled tumor cells and the Cy5 labeled siRNA@MSN after $24 \mathrm{~h}$ i.v. injection. The green signals indicated the orthotopic colorectal tumor and the red fluorescence signals denoted the Cy5-labeled 
nanoparticles. The nanoparticle distribution in the tumors observed from the perspective, coronal, sagittal, and transaxial directions were separately shown. (C) Treatment regimen. (D, H) In vivo bioluminescence imaging of HCT116-luc (D) and COLO205-luc $(\mathrm{H})$ tumor at the indicated time. $(\mathrm{E}, \mathrm{I})$ Tumor growth profiles obtained through quantifying the bioluminescence in panel D and $\mathrm{H}$. (a) siPum1@MSN versus saline or siNC@MSN. (b) siPum1@MSN versus siPum2@MSN. (c) siPum1/2@MSN versus all other groups. (F, J) Tumor weight on the last day (day 30 ) of the test. $(G, K)$ Mice body weight. The data are expressed as mean \pm s.d. $(n=5)$. ${ }^{\star} P<0.05,{ }^{\star} \mathrm{P}<0.01,{ }^{\star \star \star} P<0.001$.

\section{Supplementary Files}

This is a list of supplementary files associated with this preprint. Click to download.

- TableS1Primers.xlsx

- TableS2PUM1KOCHANGEDmRNA.xIsx

- TableS3PUM2KOCHANGEDmRNA.xIsx

- TableS4PUM1KOUPprotein.xlsx

- TableS5PUM2KOUPprotein.xlsx

- TableS6PUM1PARCLIP.xlsx

- GongetalSupplementaryFiguresNatureCommunications20210202.docx 